\title{
Neurological Complications of Anderson-Fabry Disease
}

\author{
Antonino Tuttolomondo ${ }^{1 *}$, Irene Simonetta ${ }^{1}$, Salvatore Miceli $^{1}$, Valentina Arnao ${ }^{2}$, Giuseppe Licata ${ }^{1}$ and \\ Antonio Pinto ${ }^{1}$
}

${ }^{1}$ Dipartimento Biomedico di Medicina Interna e Specialistica, Università degli Studi di Palermo (Italy); ${ }^{2}$ Dipartimento di Biopatologia e Neuroscienze Cliniche, Università degli Studi di Palermo, Italy

\begin{abstract}
Characteristic clinical manifestations of AFD such as acroparesthesias, angiokeratoma, corneal opacity, hypo/ and anhidrosis, gastrointestinal symptoms, renal and cardiac dysfunctions can occur in male and female patients, although heterozygous females with AFD usually seem to be less severely affected. The most prominent CNS manifestations consist of cerebrovascular events such as transient ischaemic attacks (TIAs) and (recurrent) strokes. For the most part, CNS complications in AFD have been attributed to cerebral vasculopathy, including anatomical abnormalities.

The natural history of Fabry patients includes transitory cerebral ischaemia and strokes, even in very young persons of both genders. The mechanism is partly due to vascular endothelial accumulation of Gb-3. White matter lesions (WML) on occur MRI. Both males and females can be safely treated with enzyme replacement; and thus screening for Fabry disease of young stroke populations should be considered. There are, however, no hard data of treatment effect on mortality and morbidity. Stroke in Anderson-Fabry disease study of 721 patients with cryptogenic stroke, aged 18-55 years, showed a high prevalence of Fabry disease in this group: 5\% (21/432) of men and 3\% (7/289) of women. Combining results of both sexes showed that $4 \%$ of young patients with stroke of previously unknown cause had Fabry disease, corresponding to about 1-2\% of the general population of young stroke patients. Cerebral micro- and macro-vasculopathy have been described in Fabry disease. Neuronal globotriaosylceramide accumulation in selective cortical and brain stem areas including the hippocampus has been reported by autopsy studies in FD, but clinical surrogates as well as the clinical relevance of these findings have not been investigated so far. Another Neurologic hallmark of Fabry disease (FD) includes small fiber neuropathy as well as cerebral micro- and macroangiopathy with premature stroke. Cranial MRI shows progressive white matter lesions (WML) at an early age, increased signal intensity in the pulvinar, and tortuosity and dilatation of the larger vessels. Conventional MRI shows a progressive load of white matter lesions (WMLs) due to cerebral vasculopathy in the course of FD. Another study has been conducted to quantify brain structural changes in clinically affected male and female patients with FD. The peripheral neuropathy in Fabry disease manifests as neuropathic pain, reduced cold and warm sensation and possibly gastrointestinal disturbances. Patients with Fabry disease begin having pain towards the end of the first decade of life or during puberty. Children as young as 6 years of age have complained of pain often associated with febrile illnesses with reduced heat and exercise tolerance. The patients describe the pain as burning that is often associated with deep ache or paresthesiae. Some patients also have joint pain. A high proportion of patients with Fabry disease is at increased risk of developing neuropsychiatric symptoms, such as depression and neuropsychological deficits. Due to both somatic and psychological impairment, health-related quality of life $(\mathrm{QoL})$ is considerably reduced in patients with Fabry disease.
\end{abstract}

Targeted screening for Fabry disease among young individuals with stroke seems to disclose unrecognized cases and may therefore very well be recommended as routine in the future. Furthermore, ischemic stroke is related to inflammation and arterial stiffness and no study had addressed this relationship in patients with AF disease and cerebrovascular disease, so this topic could represent a possible future research line.

Keywords: ???????????????????????????????????????????.

\section{INTRODUCTION}

Anderson-Fabry Disease (AFD) is a rare genetic X-linked disorder with deficient activity of the lysosomal enzyme alphagalactosidase A [1]. The resultant progressive and diffuse accumulation of neutral glycosphingolipids, especially of globotriaosylceramide (Gb3) in vascular endothelium, kidneys, heart, brain, skin, cornea and other tissues leads to multiorgan pathology. Characteristic clinical manifestations of AFD such as acroparesthesias, angiokeratoma, corneal opacity, hypo/ and anhidrosis, gastrointestinal symptoms, renal and cardiac dysfunctions can occur in male and female patients, although heterozygous females with AFD usually seems to be less severely affected $[2,3,4]$. In the central nervous system (CNS), diffuse lipid storage occurs in the cerebral vasculature and neurons. The most prominent CNS manifestations (Table 1) consist of cerebrovascular events such as transient ischaemic attacks (TIAs) and (recurrent) strokes [5,6,7]. For the most part, CNS complications in AFD have been attributed to cerebral vasculopathy, including anatomical abnormalities (e.g. small vessel occlusive disease, and large vessel ectasia and tortuosity) [8,9],

*Address correspondence to this author at the Dipartimento Biomedico di Medicina Interna e Specialistica, Università degli Studi di Palermo, Italy; Tel:/Fax: ??????????????????; E-mail: bruno.tuttolomondo@unipa.it
Table 1. Neurological and Sensory Organ Complications of Fabry Disease (from Nervous System Manifestations of Fabry Disease: Data from FOS - the Fabry Outcome Survey, Chapter 23)

\begin{tabular}{|rl|}
\hline Central nervous system \\
\hline - & Ischaemic stroke \\
- & Haemorrhagic stroke \\
- & Epilepsy \\
- & Cognitive impairment \\
\hline Peripheral nervous system \\
\hline$\bullet$ & Painful small fibre neuropathy \\
- & Autonomic dysfunction \\
\hline Sensory organs \\
\hline & Ear - hearing loss, vertigo, tinnitus \\
- & Eye - cornea verticillata, subcapsular cataract, retinal vascular \\
& tortuosity \\
\hline
\end{tabular}




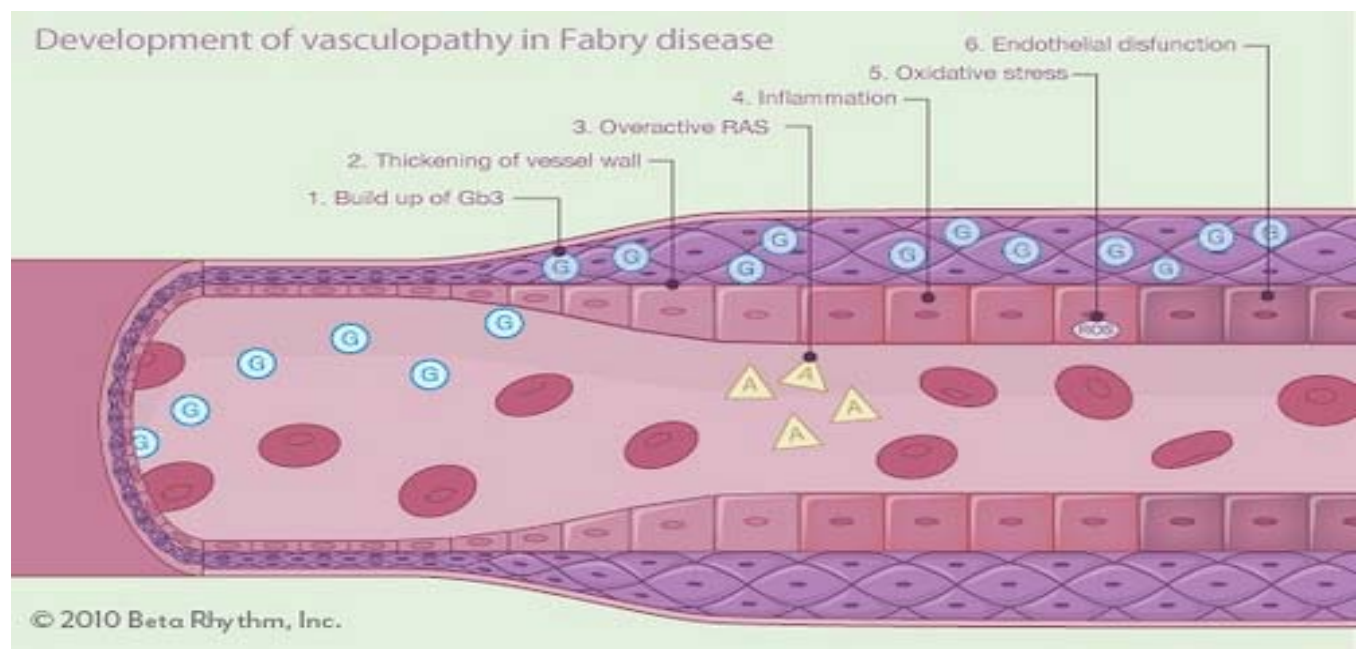

Fig. (1).

impairment of endothelial function and dysregulation of cerebral blood flow [10,11] (Fig. 1). Gb3 storages within neurons [12,13], cerebrovascular risk factors $[14,15]$ and genetic factors [16] might increase additionally the likelihood of developing cerebrovascular disease. Brain magnetic resonance imaging (MRI) results often altered in AFD patients. In detail, neuroradiological findings include periventricular white matter signal intensity abnormalities and single or multiple lacunar infarcts (alterations typical of small vessel disease), large ischemic cerebral infarctions, and posterior thalamus involvement (the so-called pulvinar sign) $[17,18]$.

\section{STROKE IN ANDERSON-FABRY DISEASE}

Stroke and transitory cerebral ischemia are two possible complications of Anderson-Fabry disease and there complications are due to to vascular endothelial accumulation of $\mathrm{Gb}-3$, so on this basis these neurological complication are a clinical expression of a brain vessel disease causing lacunar lesions or larger lesions or white matter lesions (WML) on MRI. The analyses of results from ongoing studies will add to the decision on whether or not to screen young stroke patients for Fabry disease. Finally, stroke prophylactic therapy should be used liberally in patients of both genders with verified Fabry disease. This includes primary prevention such as lifestyle counseling, targeting blood pressure, managing atrial fibrillation, diabetes mellitus, hyperlipidaemia, and ASA.

Stroke in young adults is etiologically diverse and may represent a diagnostic challenge remaining cryptogenic in one-fourth of cases. Limited information is available on the prevalence of Fabry's disease, a treatable multisystem inherited lysosomal storage disorder, and disability in young patients with cryptogenic stroke. The Canadian Fabry Stroke Screening Initiative (CFSSI) [19] is a prospective multicenter cohort study of young adults (age 18-55) presenting with an ischemic stroke, transient ischemic attack, or intracerebral haemorrhage of unknown etiology to stroke centres across Canada. Diagnosis of Fabry's disease is made by direct DNA analysis of blood samples for $\alpha$-galactosidase gene mutations or polymorphisms. Demographics, clinical information, and investigations, including brain Magnetic Resonance Imaging (MRI), are collected. Functional neurological assessment includes neurological examination, the National Institutes of Health (NIH) stroke scale, modified Rankin scale, and the Barthel index. A follow-up interview is conducted by telephone or in person approximately six-months after the index stroke/transient ischemic attack/intracerebral haemorrhage to determine patient outcomes, quality of life, and patient use of medications. Prevalence of positive DNA mutation or single nucleotide polymorphism screens for Fabry's disease as a propor- tion of total cryptogenic stroke. Secondary outcomes include incident risk of new or recurrent vascular event at six-months, discharge disposition, disability at six-months as measured by the modified Rankin scale, mean time from symptoms onset to the definite etiological diagnosis, and length of hospital stay. This study constitutes the first initiative to determine the prevalence of a positive screen for Fabry's disease in young adults with stroke in Canada. Moreover, the Canadian Fabry Stroke Screening Initiative will provide information on recurrent vascular events, disability at sixmonths (modified Rankin scale), and disposition in this understudied population.

A recent study conducted in 721 patients with cryptogenic stroke showed a high prevalence of Fabry disease in this group. In particular this study showed that $5 \%$ of male subjects with cryptogenic stroke and 3\% of women had AFD [20] (Table 2).

These findings make AFA another potential cause of cryptogenic stroke such as cardiogenic embolism, atherosclerotic vasculopathy, and nontraumatic cervico-cerebral arterial dissection $[21,22,23]$.

Another study showed that in young stroke patients with proven Fabry disease, WML load was clearly higher than in patients with other causes of cryptogenic stroke without evidence for Fabry disease [20].

Rolfs and co-workers also showed in strokes patients with AFD disease had more frequently posterior strokes (vertebrobasilar) than in the carotid circulation.

A very recent study conducted by Sarikaya et al. [24] aimed to evaluate the frequency of unrecognized FD in a cohort of stroke patients at a tertiary stroke center. Patients suffering from first cryptogenic ischaemic stroke or transient ischaemic attack (TIA) at the age of 18-55 years were screened for the presence of FD. Authors measured the serum activity of $\alpha$-galactosidase ( $\alpha$-GAL) in all patients. In addition, sequencing of $\alpha-\mathrm{GAL}$ gene was performed in men with low enzyme activity and in all women. They have recruited 150 patients. The $\alpha$-GAL activity was low in nine patients (6\%; six men and three women). Genetic sequencing in six men with low enzyme activity and all 48 women detected no $\alpha$-GAL gene mutation. This study suggests that the yield of screening for FD in patients with first cryptogenic ischaemic stroke or TIA is very low. Further large-scale studies are needed to investigate the importance of FD amongst patients with recurrent cryptogenic strokes. Nevertheless, the cause of initial ischemic stroke in up to $30 \%$ of young patients remains unclear. Some authors hypothesized that alpha-Gal A deficiency is a rare cause of initial early-onset 
Table 2. Cerebrovascular and common clinical features in young adult patients with cryptogenetic stroke with and without Fabry disease

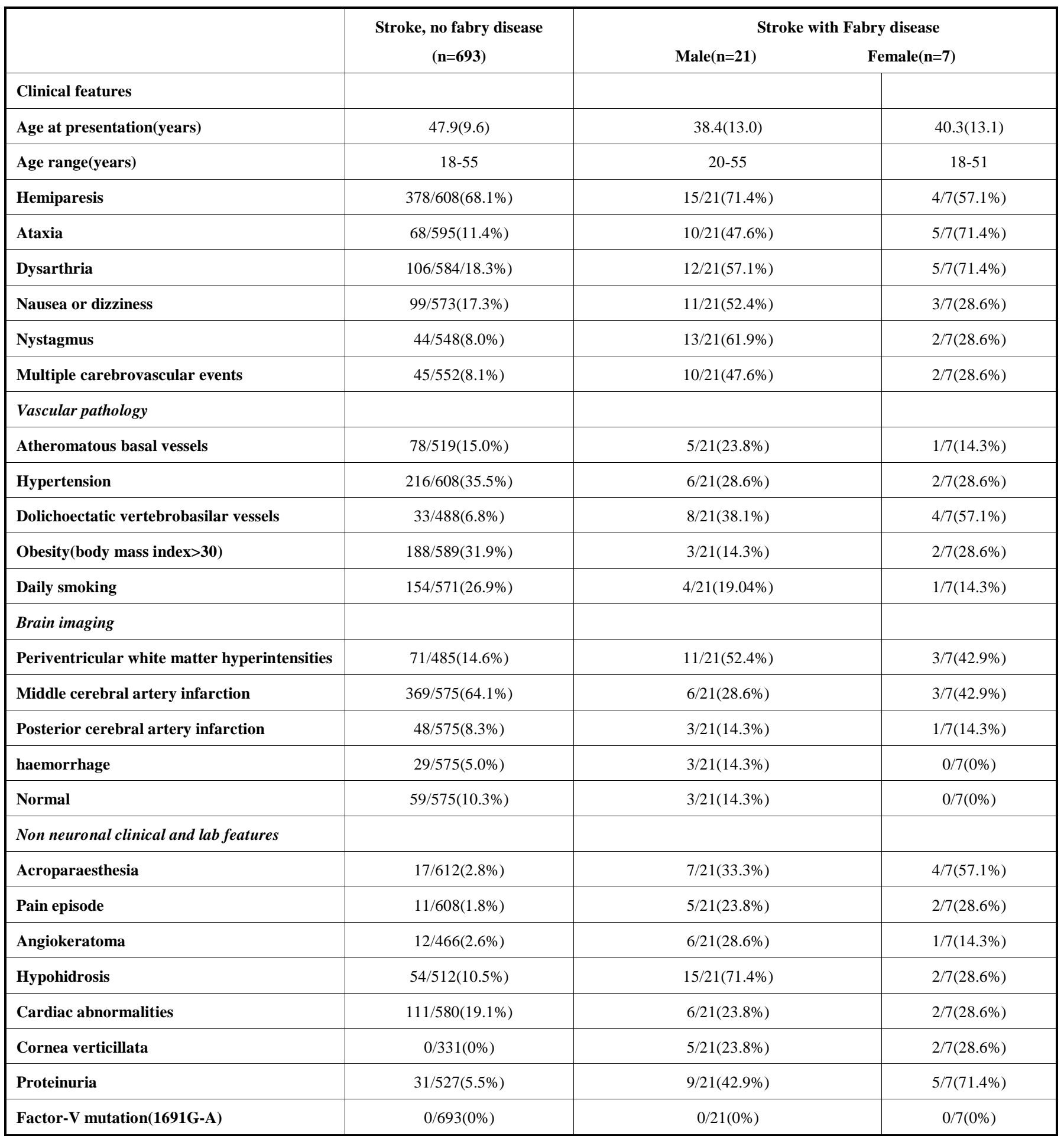

Data are mean(SD)of number $(\%)$. $* \mathrm{P}<0.05$

ischemic stroke in men. The Stroke Prevention in Young Men Study [25] enrolled about 550 men (15 to 49 years) with first ischemic stroke in the Baltimore-Washington area in 2004 to 2007. Frozen plasma samples were assayed for alpha-Gal A activity, and DNA from patients with consistently low plasma alpha-Gal A activities were sequenced. The study sample consisted of 558 men (42\% African-American; median age 44 years). Stroke was cryptogenic in 154 men (40\% African-American). In 10 patients with low plasma alpha-Gal A activities, DNA sequencing identified altera- tions in the alpha-Gal A gene in 2 patients. The polymorphism, D313Y, which results in low plasma enzyme activity, but near normal levels of cellular activity was seen in one EuropeanAmerican male. The Fabry disease-causing A143T mutation was seen in an African-American male with cryptogenic stroke $(0.18 \%$ of all strokes: upper $95 \% \mathrm{CI}=0.53 \% ; 0.65 \%$ of cryptogenic strokes: upper $95 \% \mathrm{CI}=1.92 \%$ ). In this biracial population, unrecognized Fabry disease is a rare but treatable cause of initial ischemic stroke in young men (Tables $\mathbf{3 , 4}$ ). 
Table 3. Prevalence of Fabry disease in young men with cryptogenic ischemic stroke.

\begin{tabular}{|c|c|c|c|c|c|c|}
\hline \multicolumn{3}{|c|}{ First Ischemic Stroke } & \multicolumn{3}{c|}{ Recurrent Ischemic Stroke } \\
\hline & Total No. & No. With Fabry Disease & Prevalence & Total No. & No. With Fabry Disease & Prevalence \\
\hline \hline Rolfs $^{*}$ & 367 & 8 & 2.17 & 41 & 10 & 24.3 \\
\hline Brouns & 46 & 0 & 0 & 9 & 0 & 0 \\
\hline Wozniak & 154 & 1 & 0.65 & N/A & N/A \\
\hline
\end{tabular}

*Estimated from reference ${ }^{20}$ using the following 4 assumptions: (1) total of 432 men with any stroke, assuming the same rate of hemorrhage as entire sample (5.5\%), then 408 men with any ischemic stroke; (2) assuming the same rate of recurrent stroke as in the entire sample (10\%), then 367 men had an initial ischemic stroke; (3) 10 men with Fabry disease had recurrent stroke and 11 with initial stroke; (4) given the high mortality rate of hemorrhage, assuming that 3 men with Fabry disease and hemorrhagic stroke were all initial strokes, then 8 men with Fabry disease had initial ischemic stroke $†$ Dr R. Brouns and personal communication (Dr R. Brouns, 2009).

Table 4. The Summary of Mutation Analysis in Patients with Consistently Low a-Gal A Activity.

\begin{tabular}{|c|c|c|c|}
\hline$\alpha$-Gal A Activity & & & \\
\hline \multirow[t]{4}{*}{ Initial Assay $^{\ddagger}$} & Confirmatory Assay & Male Patients With Stroke & Mutation Analysis \\
\hline & & 1 & A143T \\
\hline & & 2 & $\mathrm{WT}^{*}$ \\
\hline & & 3 & WT \\
\hline 2.08 & 1.00 & 4 & WT \\
\hline 4.62 & 6.80 & 5 & D313Y \\
\hline 2.84 & 3.09 & 6 & WT \\
\hline 2.03 & 2.23 & 7 & WT \\
\hline 0.71 & 0.36 & 8 & WT \\
\hline 2.94 & 3.20 & 9 & WT \\
\hline 4.06 & 2.69 & 10 & WT \\
\hline 1.01 & 1.66 & & \\
\hline 3.04 & 1.67 & & \\
\hline 3.75 & 3.50 & & \\
\hline
\end{tabular}

*Wild type.

$\dagger \mathrm{nmol} / \mathrm{hr} / \mathrm{mL}$ protein; normal mean \pm SD: $15.6 \mathrm{nmol} / \mathrm{hr} / \mathrm{mL}$ protein; normal mean=same for all patients.

\$All values are means of duplicate enzyme assays

The prevalence of Fabry disease, as reported by the study by Rolfs and coworkers, in young patients with cryptogenic stroke was reported to be as high as $4.9 \%$ in men and $2.4 \%$ in women [20]. This finding, however, was not reproduced in a smaller, retrospective study [26]. White matter lesions and vertebrobasilar dolichoectasia are commonly found in patients with Fabry disease [27], but literature data on the prevalence of Fabry disease in patients with these neuroimaging findings are nonexistent.

On this basis the aim of the Belgian Fabry Study (BeFaS) [28] was to prospectively assess the frequency of Fabry disease in young Belgian patients with neurologic hallmarks of this disease, namely, stroke, unexplained white matter lesions, and vertebrobasilar dolichoectasia (Fig. 2). In this national, prospective, multicenter study, authors screened for Fabry disease in 1000 patients presenting with ischemic stroke, transient ischemic attack, or intracranial hemorrhage; unexplained white matter lesions; or vertebrobasilar dolichoectasia. In male patients, they measured alpha-galactosidase A
(alpha-GAL A) activity in dried blood spots. Female patients were screened for mutations by exonic DNA sequencing of the alphaGAL A gene. Alpha-GAL A activity was deficient in 19 men (3.5\%), although all had normal alpha-GAL A gene sequences. Enzymatic deficiency was confirmed on repeat assessment in 2 male patients $(0.4 \%)$. Authors identified missense mutations in 8 unrelated female patients (1.8\%): Asp313Tyr ( $=5)$, Ala143Thr $(n=2)$, and Ser126Gly $(n=1)$. The pathogenicity of the 2 former missense mutations is controversial. Ser126Gly is a novel mutation that can be linked to late-onset Fabry disease. Alpha-GAL A deficiency may play a role in up to $1 \%$ of young patients presenting with cerebrovascular disease. These findings suggest that atypical variants of Fabry disease with late-onset cerebrovascular disease exist, although the clinical relevance is unclear in all cases.

A study [29] was undertaken to better understand the natural history of cerebrovascular manifestations of Fabry disease. Data from 2446 patients in the Fabry Registry were analyzed to identify 


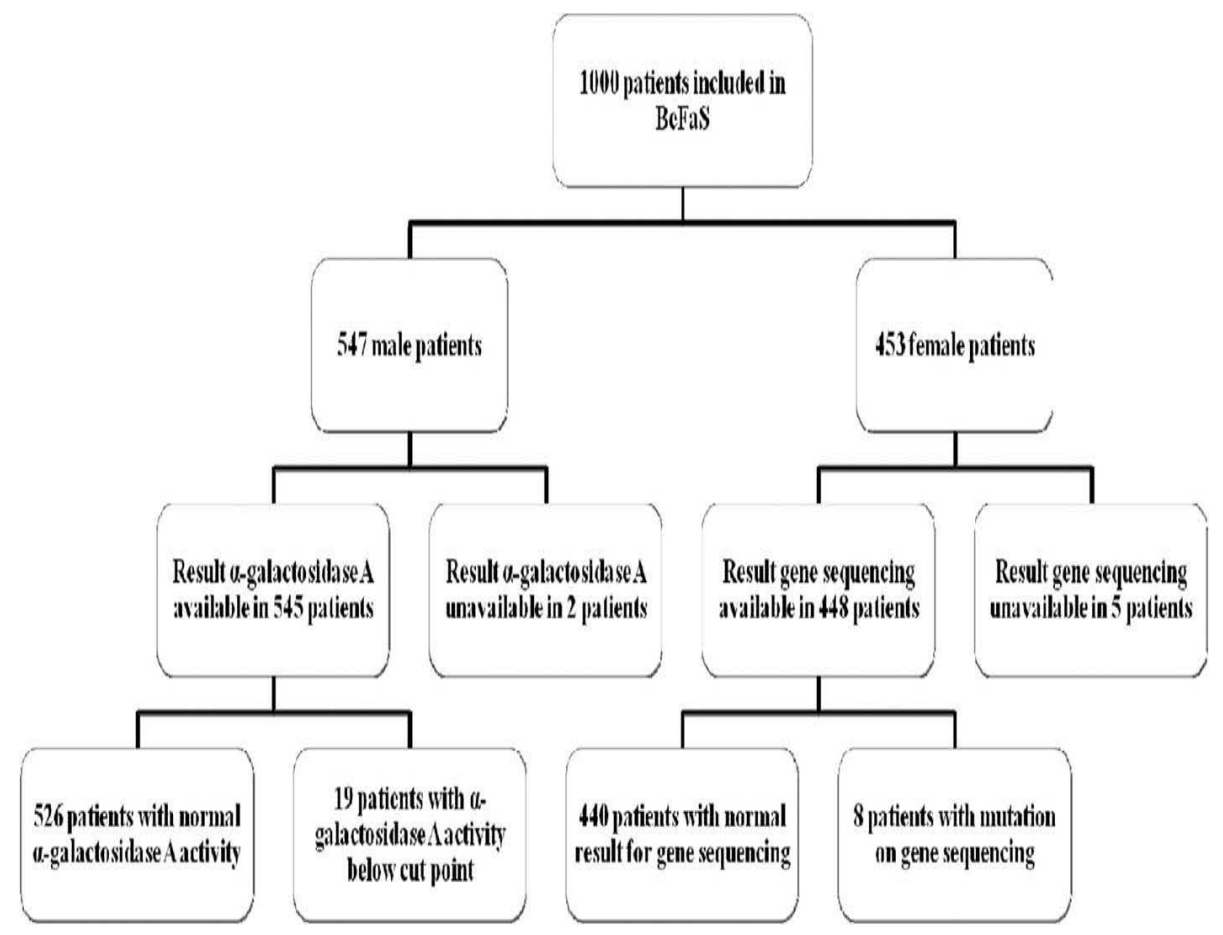

Fig. (2). Flowchart for the BeFaS.

[Brouns R et al. Stroke 2010]

clinical characteristics of patients experiencing stroke during the natural history period (ie, before enzyme replacement therapy). A total of 138 patients [86 of 1243 males $(6.9 \%)$ and 52 of 1203 females $(4.3 \%)$ ] experienced strokes. Median age at first stroke was 39.0 years in males and 45.7 years in females. Most patients $(70.9 \%$ of males and $76.9 \%$ of females) had not experienced renal or cardiac events before their first stroke. Fifty percent of males and $38.3 \%$ of females experienced their first stroke before being diagnosed with Fabry disease. Thirty patients ( 21 males and 9 females) had strokes at age $<30$ years. Most patients $(86.8 \%)$ had ischemic strokes, but $16.9 \%$ of males and $6.9 \%$ of females had hemorrhagic strokes, among those for whom stroke type was reported. At the most recently available follow-up examination after their first stroke, $60 \%$ of males and $25.5 \%$ of females exhibited stage 3 to 5 chronic kidney disease and $66.1 \%$ of males and $59.5 \%$ of females had left ventricular hypertrophy. All patients with Fabry disease, regardless of age or gender, should be monitored for possible cerebrovascular complications, as stroke can occur in the absence of other key signs of the disease.

Cerebral micro- and macro-vasculopathy have been described in Fabry disease. Neuronal globotriaosylceramide accumulation in selective cortical and brain stem areas including the hippocampus has been reported by autopsy studies in FD, but clinical surrogates as well as the clinical relevance of these findings have not been investigated so far. Some authors [30] measured the hippocampus volumes in a group of clinically affected patients with FD and correlated the findings with the cognitive performance of the patients. Hippocampal volumes were determined manually on T1-weighted MR-images of 25 FD patients (age $36.5 \pm 11.0$ years) and 20 agematched controls. Additionally, individual white matter (WM) and gray matter (GM) volumes were measured using brain segmentation analyses. After controlling for age, white matter lesion (WML) volume, WM/GM-volumes and hippocampal volumes were significantly decreased in FD. These findings were substantially more pronounced in a subgroup of men with FD. WM and WM/GM volumes, and memory function did not significantly differ between patients and controls. In patients with FD hippocampal volumes were neither significantly correlated to WML volume nor to WM or WM/GM volumes. Hippocampus atrophy was not driven by the WML or other brain tissue atrophy and seems to correlate with the neuronal involvement in FD. In this young to middle-aged Fabry cohort the hippocampus degeneration was functionally compensated without memory impairment. Longitudinal studies are needed to determine whether this degenerative component in FD will progress and, in concert with the individual WML-load, predict subsequent cognitive decline.

\section{CEREBRAL INVOLVEMENT IN ANDERSON-FABRY DIS-} EASE

White matter lesions (WML) and increased signal intensity in the pulvinar, and tortuosity and dilatation of the larger vessels (Fig. 3) are typical neuroimaging findings of SNS involvement in Anderson-Fabry disease.

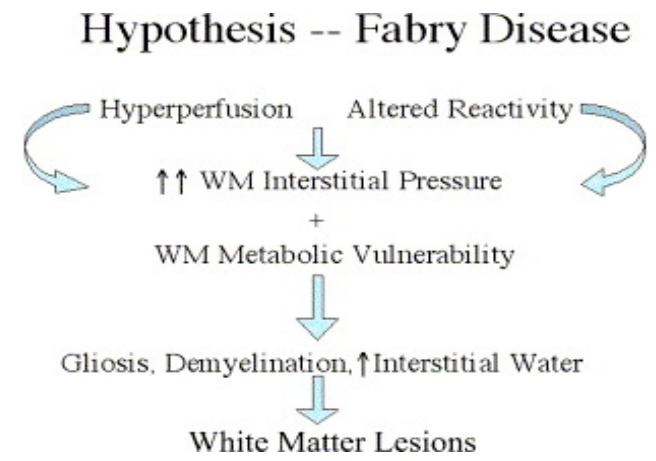

Fig. (3). Schematic hypothesized mechanism of the development of WML in Fabry disease. WM: white matter. [From David F. Moore et al. White matter lesions in Fabry disease occur in 'prior' selectively hypometabolic and hyperperfused brain regions. Brain Research Bulletin Volume 62, Issue 3, 30 December 2003] 
Fellgiebel et al. [31] compared the diagnostic utility of the different MR imaging findings in patients with AFD. These authors evaluated MRI, MR angiography, and diffusion tensor imaging (DTI). Authors showed that $87 \%$ of the individuals were correctly classified by basilar artery diameters (sensitivity 95\%, specificity 83\%) whereas WML volumes and global MD values did not significantly separate patients from controls. On this basis, basilar artery diameters appair the most accurate neuroimaging finding to separate patients with Fabry disease (FD) from controls. Future studies should adopt basilar artery measurements for early detection and monitoring of brain involvement in FD. Moreover, further investigations should reveal if the dilated vasculopathy in FD could be a screening marker to detect FD in a cohort of other cerebrovascular diseases, especially in cryptogenic stroke.

Cerebral manifestations that might be mainly due to progressive cerebrovascular dysfunction, are one major and often life-threatening burden of the disease. In female FD patients who were considered to be less affected "carriers" for a long time, the prevalence of cerebrovascular events seems to be at last as high as in male patients. In structural imaging white matter lesions (WML) can be found frequently even in young FD patients. Clinically equally affected men and women with FD showed a comparable severity of WML load. Different pathophysiological aspects of cerebral angiopathy and WML development are discussed against the background of current concepts (e. g. accumulation of Gb3 in vascular endothelium with consecutive cell proliferation and luminal stenosis, acceleration of focal intravasal pressure and disturbances of vascular auto-regulation). Pathological increase of pulvinar signal in T1-weighted MRI has also been described in FD. This finding was assumed to be caused by calcification as a consequence of disturbed local circulation. To enhance our knowledge about the relevant neurobiological processes the authors propose a more sensitive and early detection of brain structural changes in FD. New brain structural MRI methods such as diffusion-tensor imaging could provide a pattern of ultrastructural changes even in young patients without visible WML. This strategy could be as well useful for quantification of possible effects of the enzyme replacement therapy on brain structural alterations in FD. Based on recent data a systematic FD-screening by measuring Gb3 in urine of young patients with cryptogenic stroke should be discussed. Basically in such cases FD should be clinically considered.

Conventional MRI shows a progressive load of white matter lesions (WMLs) due to cerebral vasculopathy in the course of FD. Another study has been conducted [32] to quantify brain structural changes in clinically affected male and female patients with FD. Authors performed a prospective Diffusion-Tensor Imaging (DTI) study in 27 adult Fabry patients $(13 \mathrm{~m}, 14 \mathrm{f})$ and 21 age-matched controls (12 m, 9f). Global Mean Diffusivity (MD) was increased in FD $(\mathrm{P}=0.003)$ whereas global Fractional Anisotropy (FA) did not differ significantly between FD and controls. Even FD patients without significant WMLs (9m, 9f) showed increased global MD (P $=0.004)$. Regions of interest with significant MD elevations were located in the frontal, parietal and temporal white matter. No differ- ences of thalamic and hippocampal DTI measurements could be detected between FD and controls. DTI parameters did not differ between male and female patients. The data provide the first evidence of a pattern of marked structural brain tissue alterations in adult FD male and female patients even without WMLs. DTI seems to be an appropriate diagnostic tool to quantify brain tissue integrity in FD. Moreover, this method could be favorable for longitudinal assessment of brain structure alterations in FD, and for monitoring the cerebral effects of enzyme replacement therapy.

Conventional MRI shows an extensive load of white matter lesions (WMLs) which may already be present at an early stage in the disease. Albrecht et al. [33] investigator/ independent and sensitive quantification of structural changes in the brain in clinically affected men and women with FD (Table 5). Authors performed a voxel based analysis of diffusion tensor images (DTI) in 25 patients with FD and 20 age matched normal controls. DTI revealed significant increases in cerebral white matter mean diffusivity (MD) in patients with FD, which were pronounced in the periventricular white matter. Even the subgroup of patients without significant WMLs load $(n=18)$ showed increased diffusivity in the cerebral white matter. In gray matter areas, MD elevation was detected only in the posterior part of the thalamus, independent of the visible pulvinar alterations on T1 weighted images. Voxel based fractional anisotropy measurements did not differ significantly between patients and controls (Fig. 4).

The present study demonstrates the clinical feasibility of voxel based analysis of DTI as a sensitive tool to quantify brain tissue alterations in FD. The pattern of increased brain tissue diffusivity is probably due to microangiopathic alterations, mainly affecting the long perforating arteries.

\section{PERIPHERAL NERVE INVOLVEMENT IN AFD}

The peripheral neuropathy is a typical and early clinical finding of Anderson-Fabry disease (Fig. 5). Although only up to $80 \%$ of Fabry patients develop neuropathic pain, they mostly will do so by age 20 years. Also female subjects may develop neuropathic pain $[34,35]$.

The neuropathy of Fabry disease is associated with increased or immeasurable cold and warm detection thresholds in patients that are significantly different from controls in the hand, and foot[36]. Fabry patients also have reduced tolerance to a cold challenge [37].

The mechanism of AFD neuropathy is not well understood. Among possible causes a vasa nervorum glycolipid accumulation disease causing ischemia of nerves. Peripheral nervous system involvement affect mainly small A delta and C fibers; it is caused by a deficiency of alpha-galactosidase A and accumulation of alpha-Dgalactosyl moieties, particularly globotriosylceramide accumulation (Gb3). These are found in Schwann cells and dorsal root ganglia together with deposits in central nervous system neurons and are likely causally related to the altered autonomic function and neuropathic pain found in this disorder.

Table 5. White Matter Lesion Quantification in Patients with Fabry Disease and in Controls

\begin{tabular}{|c|c|c|c|c|c|c|c|c|}
\hline \multirow{2}{*}{ WML classification } & \multicolumn{2}{|c|}{ Male Fabry patients } & \multicolumn{2}{c|}{ Female Fabry patients } & \multicolumn{2}{c|}{ Male controls } & \multicolumn{2}{c|}{ Female controls } \\
\cline { 2 - 9 } & $\mathbf{n}$ & Age & $\mathbf{n}$ & Age & n & Age & n & Age \\
\hline \hline No WML & 4 & $26.8(7.0)$ & 7 & $30.7(11.0)$ & 9 & $34.1(8.3)$ & 7 & $34.1(8.4)$ \\
\hline Mild WMLs & 2 & $30.0(4.0)$ & 5 & $39.4(9.6)$ & 3 & $35.3(17.1)$ & 1 & 49.0 \\
\hline Significant WMLs & 4 & $48.5(5.7)$ & 3 & $46.0(10.1)$ & 0 & & 0 \\
\hline
\end{tabular}




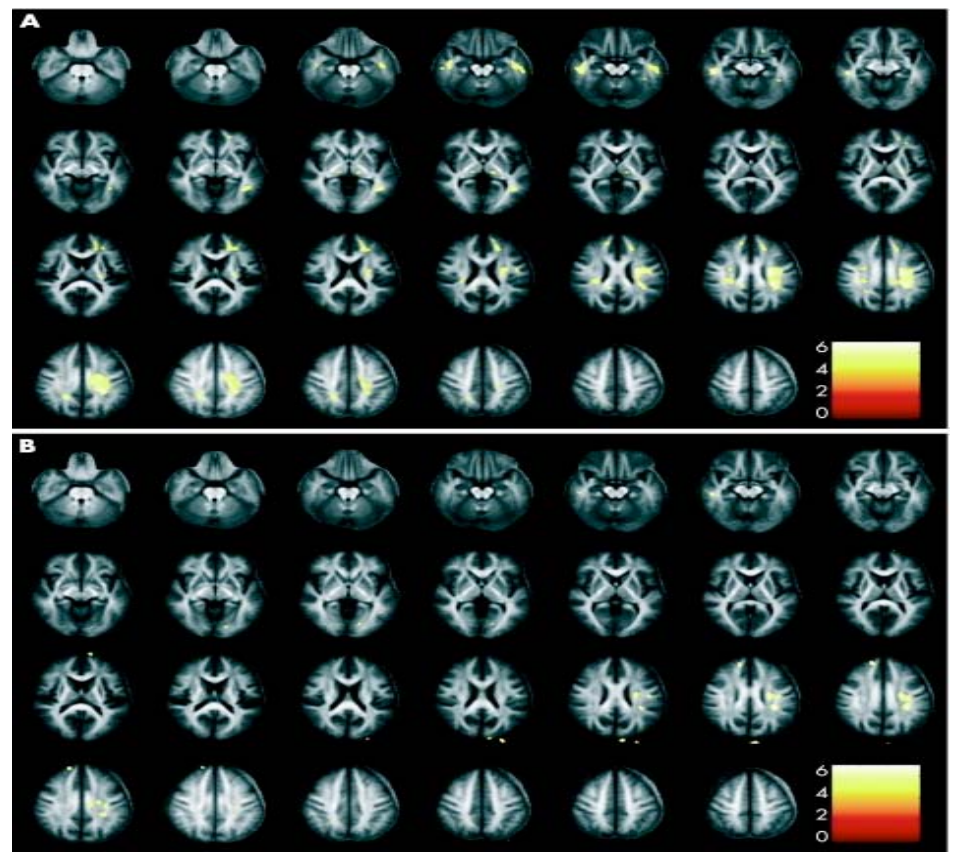

Fig. (4). Voxel based comparison of mean diffusivity (MD) between patients with Fabry disease (FD), with (A) and without (B) significant white matter lesions (WMLs), and age matched controls. Significant MD elevations are projected on transverse sections (3 mm distance) of the fractional anisotropy template. (A) MD values were significantly increased mainly in regions of the frontal, temporal, central and parietal white matter ( $\mathrm{p}<0.001$, uncorrected). In gray matter regions, significant MD increases were detected in the posterior thalamus bilaterally. (B) FD patients without significant WMLs ( $\mathrm{n}=18)$ showed significant MD increases in similar locations but with a lower total cluster size.
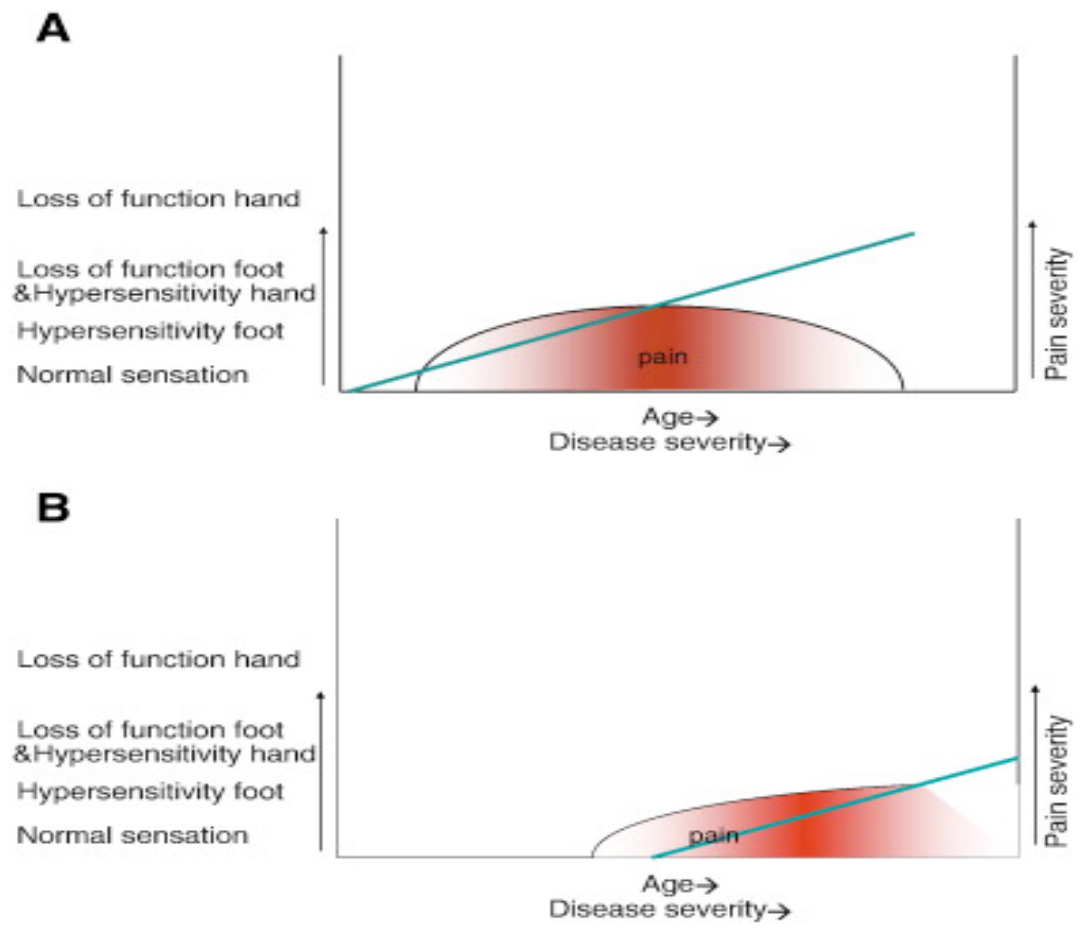

Fig. (5). a) Pain and nerve fiber dysfunction in males with Fabry disease. Red area = course of pain. Green line $=$ small nerve fiber dysfunction; with older age and more severe disease, nerve fiber dysfunction increases. b) Pain and nerve fiber dysfunction in females with Fabry disease. Red area $=$ course of pain. Green line $=$ small nerve fiber dysfunction; with older age and more severe disease, nerve fiber dysfunction increases. [From Marieke Biegstraaten et al. Small fiber neuropathy in Fabry disease. Molecular Genetics and Metabolism. June 2012].

Other related abnormalities to be discussed are hypohidrosis and other abnormalities attributed to although usually in the normal range, compared to controls, nerve conduction parameters are significantly impaired [38].
The function of the peripheral nervous system is somewhat improved by ERT with reduction in neuropathic pain and an improvement of the detection threshold for cold and warm sensation in the hand and foot. Improvement in sweating and heat tolerance is 
also noted following ERT. Despite those positive results conflicting findings exist about ERT role to normalize the function of the peripheral nervous system. Shiffmann et al. [39] reported the effect of a 3-year open-label extension of a previously reported 6-month placebo-controlled enzyme replacement therapy (ERT) trial in which 26 hemizygous patients with Fabry disease received 0.2 $\mathrm{mg} / \mathrm{kg}$ of alpha-galactosidase A every 2 weeks. The effect of ERT on neuropathic pain scores while off pain medications, quantitative sensory testing, quantitative sudomotor axon reflex test (QSART), and thermoregulatory sweat test (TST) is reported. In the patients who crossed-over from placebo to ERT $(\mathrm{n}=10)$, mean pain-at-itsworst scores on a $0-10$ scale decreased (from 6.9 to 4.5 ). There was a significant reduction in the threshold for cold and warm sensation in the foot. At the 3-year time-point, pre-ERT sweat excretion in 17 Fabry patients was $0.24+/-0.33 \mathrm{microl} / \mathrm{mm}(2)$ vs. $1.05+/-0.81$ in concurrent controls $(\mathrm{n}=38)$. Sweat function improved $24-72 \mathrm{~h}$ post-enzyme infusion $(0.57+/-0.71 \mathrm{microl} / \mathrm{mm}(2)$ and normalized in four anhidrotic patients. TST confirmed the QSART results. Authors concluded that prolonged ERT in Fabry disease leads to a modest but significant improvement in the clinical manifestations of the small-fiber neuropathy associated with this disorder. QSART may be useful to further optimize the dose and frequency of ERT.

Severe neuropathic pain and hypohidrosis are important symptoms of Fabry disease, particularly in the first three decades of life. The pain is associated with a length-dependent small-fibre neuropathy that also causes a selective deficiency of cold perception. Cold exposure often accentuates the pain and worsens thermal perception. The hypohidrosis leads to poor exercise and heat tolerance. The mechanisms by which alpha-galactosidase A deficiency causes these physiological abnormalities are poorly understood. The stored glycolipid (globotriaosylceramide) may interfere with the function of cellular membrane proteins, such as ion channels, or may lead to cytotoxicity (Fig. 6). The characteristic neuropathic pain can be symptomatically treated with various types of anticonvulsant drugs, such as carbamazepine. Improvement in neuropathic pain as a primary outcome measure has been useful in demonstrating that enzyme replacement therapy is effective in improving pain-related quality of life in Fabry disease. Overall, although these findings are encouraging, they do not suggest complete normalization of the peripheral nerve function. It is possible that early treatment before irreversible axonal loss, or higher and more frequent dosing may be more effective. Alternatively, as seen also in the Fabry vascular diathesis, perhaps the infused enzyme has insufficient access to affected sensory nerves and ganglia. This hypothesis is supported by the observation of poor storage clearance in non-vascular endothelial cells in a patient who received 2.5 years of infusions of agalsidase beta. The dysfunction of the peripheral nervous system is easily assessable and more readily reversible with specific therapy than the destructive processes that occur in organs such as the kidney. In future, therefore, it is likely that neuropathic pain, quantitative sensory testing and hypohidrosis will serve as clinical outcome measures for studies of specific and effective therapies for Fabry disease.

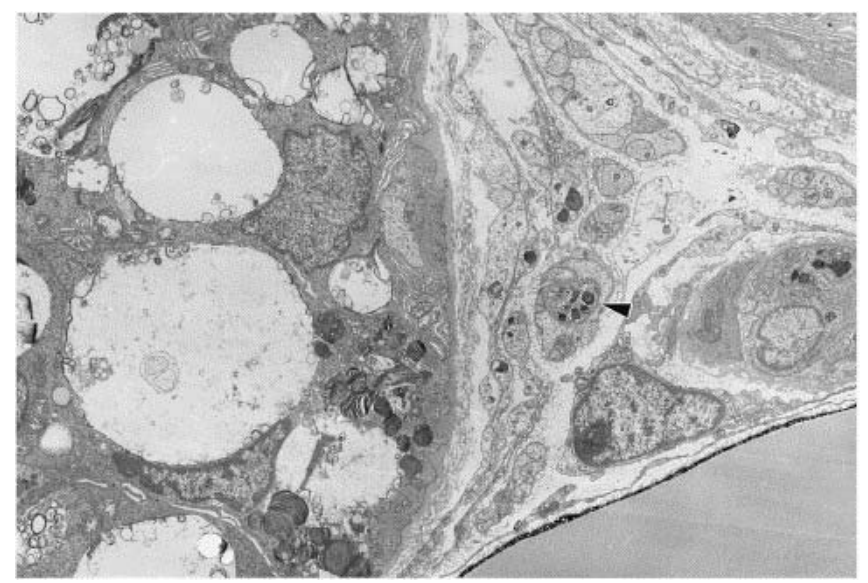

Fig. (6). Lamellated bodies (arrowhead) in the axons close to the vacuolated eccrine sweat gland $(\times 6000)$.

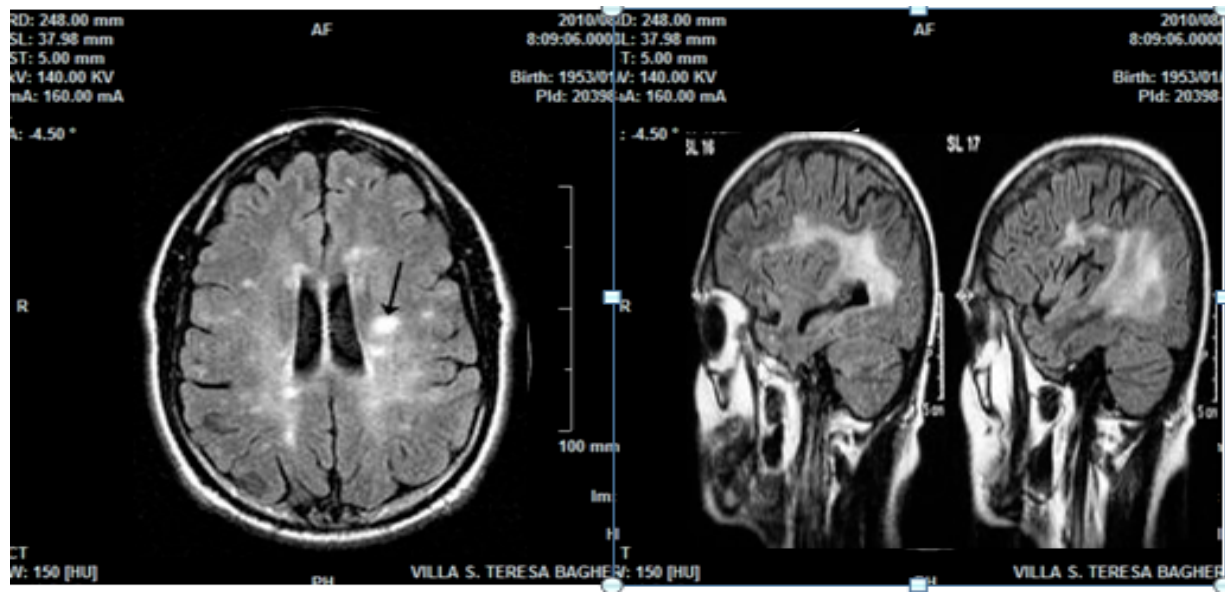

Fig. (7). Cerebral CT reveals malacic changes of periventrivular white matter, due to a probable chronic hypoxia and small hypodense areas that are compatible with ischemic lesions, in a 55 year-old female patient with AFD. 


\section{CEREBROVASCULAR INVOLVEMENT IN ANDERSON- FABRY DISEASE}

Fabry disease pathogenesis consists of a glycosphingolipid storage in endothelial and smooth muscle cells ad neurons of the autonomic nervous system (Fig. 7). Anderson-Fabry disease clinical manistestations are essentially vascular (or miscrovascular) complications (such as cerebral disease), with a not well understood pathophysiology. Some studies indicate that vascular lesions of Anderson- Fabry disease may be related to endothelial dysfunction, alterations in cerebral perfusion and a pro-thrombotic state $[40,20,41,42]$. To date not well clear is the issue about the concept if a GB3 storage in the endothelial cells and the pro-thrombotic state is the origin of arterial damage or whether smooth muscle cell proliferation in the arterial media layer could represent the first step of Anderson-Fabry vascular pathology $[43,44]$. Furthermore a small fiber neuropathy could influence vascular reactivity as well whereas normal early atherogenesis have been described in patients with AFD and various grade of organ damage. Some studies showed a high intima media thickness of various arterial sites [44,45]. This finding has been also observed in a-galactosidase A deficient mouse model [46]. Although Moore et al. [47] did not confirm this finding in a group of 7 patients with AFD.

Other studies $[48,49]$ showed that the blood flow is decreased [50] and vascular resistance is increased in patients with Fabry disease. Other authors reported a decreased coronary blood flow reserve [51,52]. Altarescu et al. showed reported an increased forearm arterial blood flow after intra-arterial infusion of acetylcholine in Anderson- Fabry patients [53] (Fig. 8). So the authors concluded that in Fabry disease the abnormal vascular responses were endothelium dependent.

In contrast, in the a-galactosidase A deficient mouse model, there was a reduced reactivity to acetylcholine [54]. Furthermore, authors showed increased activity of COX-1 and COX-2 in the same model [55]. Two clinical studies reported alterations in cerebral blood flow by transcranial ultrasonography [56,57, 58]. Nevertheless other studies reported enhanced blood flow, for example CBFV was increased in treated and untreated Fabry patients in a study by Moore et al. [59]. Studies reporting PET scanning finding confirmed this $[60,61]$ (Fig. 9).

Affected brain area topography in Anderson Fabry disease on MRI, mainly shows that the posterior and periventricular white matter, were the areas affected by a relative hyperperfusion (Fig. 10).

These findings could be due to a dysregulation of the nitric oxide pathway, leading to excessive protein nitration. White matter lesions (WMLs) are a typical finding in Fabry disease patients and they are related to microvascular ischemic lesions [62] and frequently asymptomatic.

The histopathology of Fabry arteries shows, as the most important and early finding, an involvement of the smooth muscle cell with stored glycolipid $[63,64,65]$.

Barbey et al. [65] reported that plasma of patients with AFD stimulate proliferation of vascular smooth muscle cells and cardio-
A

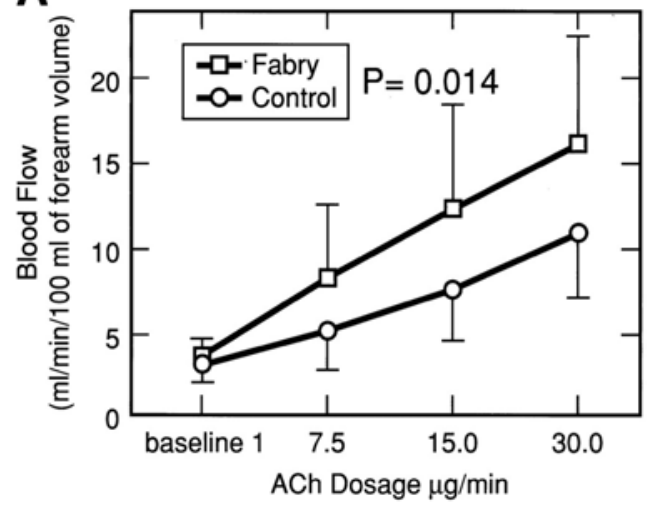

B

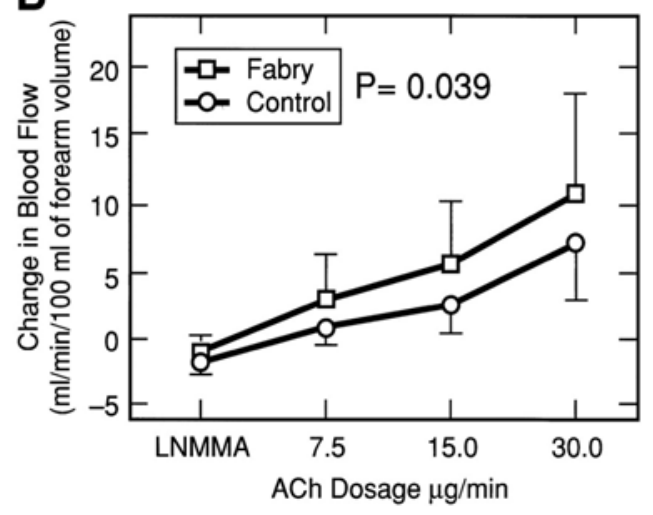

Fig. (8). Mean blood flow at baseline and after intra-arterial infusion of ACh (A) and change in mean blood flow after intra-arterial infusion of ACh and LNMMA (B)

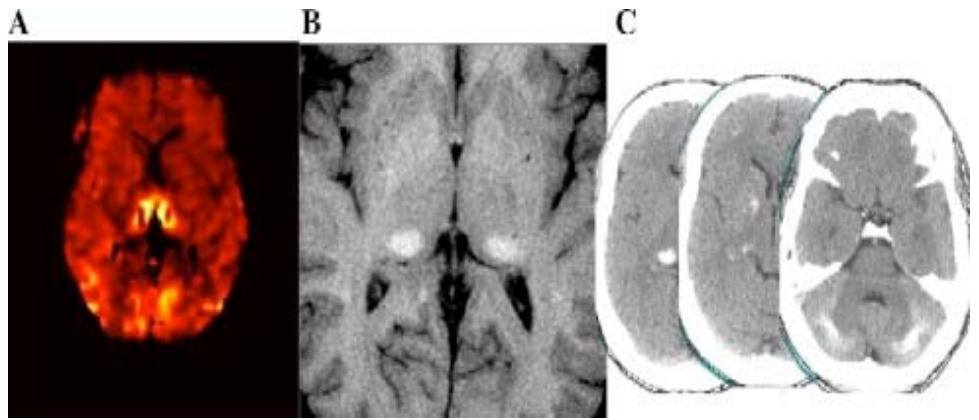

Fig. (9). A. Resting rCBF SPM $\{t\}$ map of significantly increased blood flow in Fabry group ( $\mathrm{n}=26)$ compared with control group ( $\mathrm{n}=10)$ after template coregistration of individual PET studies. Resting rCBF in control was not significantly greater than Fabry group at any set-level statistic, with no significantly corrected clusters or voxels (data no shown). Increasing SPM $\{t\}$ voxel value above threshold is shown by color bar. B. Resting $\mathrm{rCBF} S P M\{t\}$ map of significantly decreased regional cerebral blood flow in ERT patient group $(n=14)$ compared with placebo group $(n=12)$ after template coregistration of individual PET studies. No significant decrease in rCBF occurred in placebo group compared with ERT group. Increasing SPM $\{t\}$ voxel value above threshold is shown by color bar. 
myocytes, whereas Brakch et al. showed that sphingosine-1-phosphate is growth-promoting factor active in subjects in Fabry disease [66]. Furthermore Aerts et al. showed that a new substance, the lyso-compound of globotriaosylceramide (Gb3), lyso Gb3 or lyso$\mathrm{CTH}$ able to induce smooth muscle cell proliferation in vitro [67] (Fig. 11).
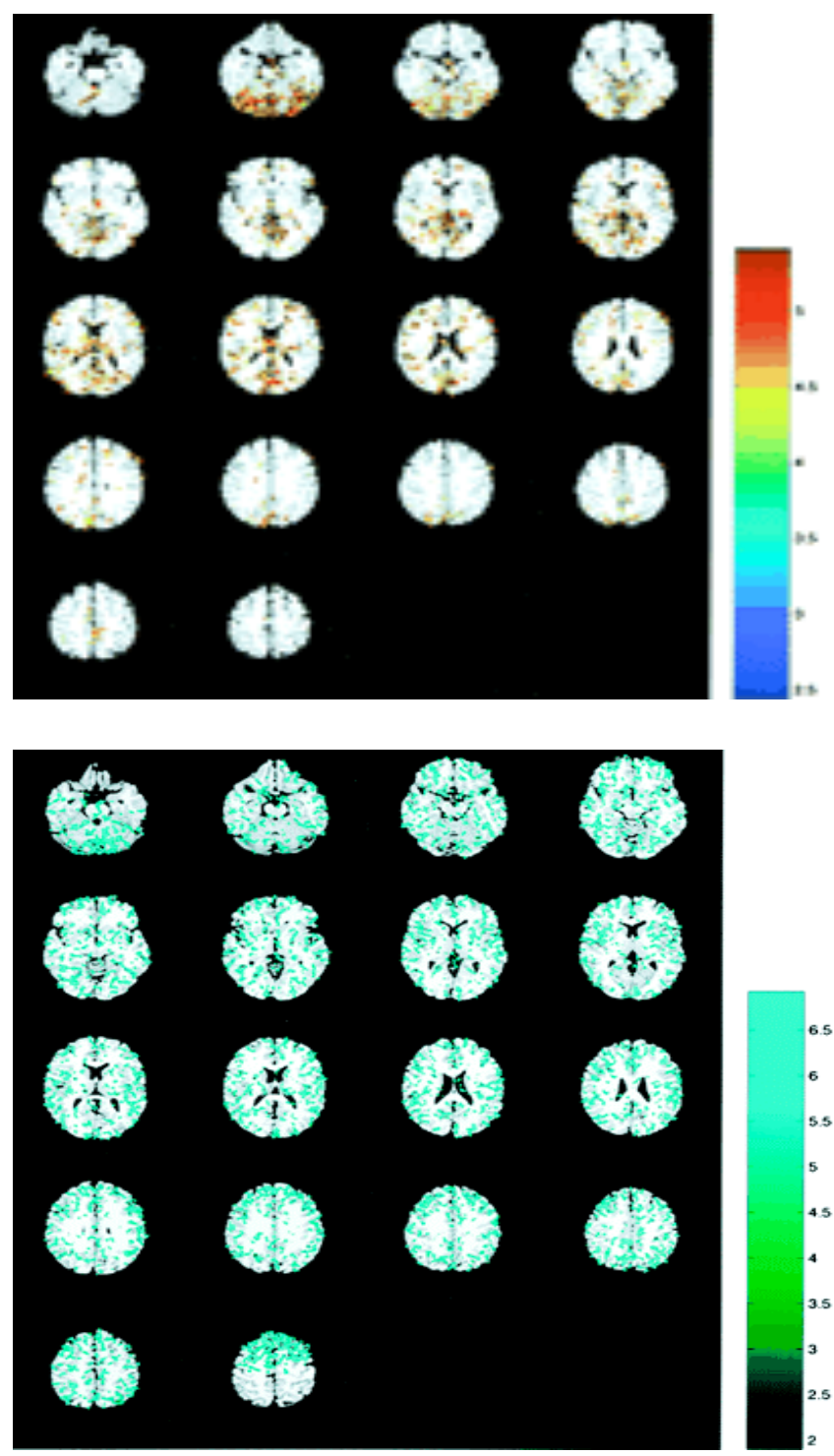

Fig. (10). End organ damage associated with cerebral hyper-perfusion. A. Cerebral blood flow map of a single patient with Fabry disease using an MRI spin tagging technique. The image shows relative hyper-perfusion in the posterior circulation territory including the pulvinar region. B. T1weighted MRI image showing pulvinar hyperintensity. This abnormality is seen in about $30 \%$ of patients with the disease. C. Cerebral CT of the Fabry patient showing calcifications in the pulvinar, cerebral white matter and cerebellum.

[From David F Moore et al. The cerebral vasculopathy of Fabry disease. Journal of the Neurological Sciences. June 2007]

The increase in IMT is probably a echographic finding more frequent among AFD alteration in vessel wall than the more traditional IMT changes observed in premature atherosclerotic disease. atheromatous plaque formation is rare in AFD and, when present, stenotic lesions have a different aspect with a more diffuse location. On this basis Fabry disease could represent a possible risk factor for widespread atherosclerosis in association with other risk factors, such as renal insufficiency, diabetes and hypertension.

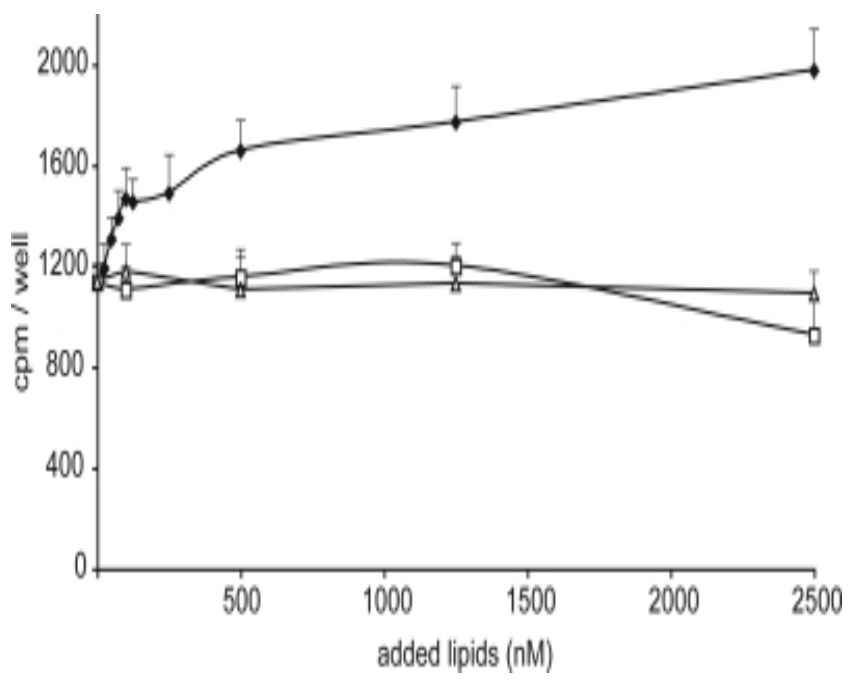

A

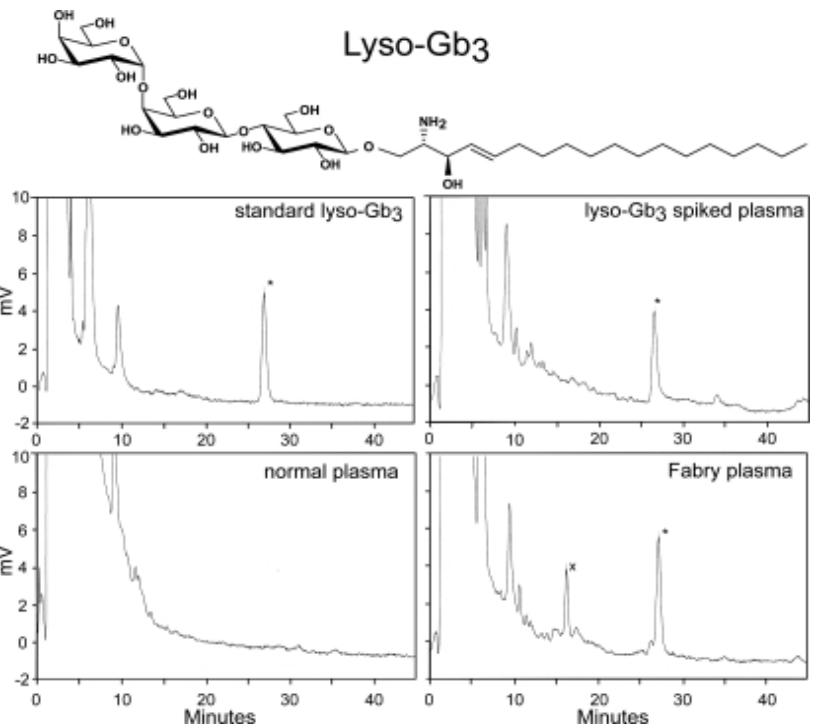

B

Fig. (11). A Detection of lyso-Gb3 in plasma of Fabry patient. (Top) Structure of lyso-Gb3. (Middle and Bottom) Chromatograms obtained for pure lyso-Gb3 (Middle Left), normal plasma (Bottom Left), normal plasma spiked with lyso-Gb3 (Middle Right), and Fabry hemizygote plasma (Bottom Right). B. Increased proliferation of smooth muscle cells by exposure to lyso-Gb3. Cells were exposed for $24 \mathrm{~h}$ to indicated concentrations of lyso-Gb3 ( ), lactosylsphingosine (口), or Gb3 ( $\Delta)$. The incorporation of radioactive thymidine per well is depicted.

Cerebrovascular disease may progress asymptomatically in the early stages of Fabry disease (FD). A recent study [68] was conducted with the aim to test whether functional transcranial Doppler (fTCD) could provide useful data in the evaluation of these presymptomatic FD patients. A cohort of 12 adult FD patients from families with the classical phenotype of the disease was evaluated with fTCD in the posterior cerebral artery. Compared to healthy controls, resting blood velocities were significantly lower in the FD cohort ( $\mathrm{p}=0.032$ for systolic, $\mathrm{p}=0.021$ for diastolic). fTCD suggested a disturbed neurovascular coupling in the visual cortex of FD patients, with lower gain $(\mathrm{p}=0.007)$ and rate time $(\mathrm{p}=0.019)$. Men had a significantly higher attenuation $(\mathrm{p}=0.013)$ and lower natural frequency $(p=0.046)$ than the heterozygous women. These data are the first to suggest that patients with FD may develop cor- 
tical vascular dysfunction in the territory of the posterior circulation, early in the natural history of the disease. If the present findings are confirmed in larger, prospective studies, fTCD will be useful for assessing stroke risk in as yet asymptomatic FD patients, improving preventive therapeutic management.

Another study by Uçeyler et al. [69] investigated cervical and cerebral blood flow characteristics in patients with Fabry disease at baseline and under enzyme replacement therapy. In this casecontrol study authors prospectively studied 68 patients with Fabry disease with extracranial and transcranial Doppler sonography and they compared extracranial and transcranial cervical and cerebral blood flow properties in all patients with Fabry disease and in subgroups of those with or without enzyme replacement therapy, male and female, and with normal or impaired renal function. Eight male patients were investigated at baseline and 1 year after initiation of enzyme replacement therapy. This study shows that cervical and cerebral blood flow parameters in patients with Fabry disease are not different from normal values regardless of sex, renal function, or enzyme replacement therapy. Cervical and cerebral blood flow measured with extracranial and transcranial Doppler sonography is not altered in patients with Fabry disease.

A study [70] evaluated cerebral blood flow velocities and cerebral autoregulation in Anderson- Fabry subjects. Authors evaluatede Resistance Index, Pulsatility Index, Cerebrovascular Resistance, and and mean CBFV Pulsatility Index, Cerebrovascular Resistance $(\mathrm{P}<0.05)$, and the transfer function gain between low frequency oscillations in mean blood pressure and mean CBFV were higher in patients than in controls. Authors showed that the decrease of CBFV may be due to stenoses of resistance vessels and dilatation of the insonated segment of the middle cerebral artery due to reduced sympathetic tone and vessel wall pathology with decreased elasticity. Both, reduced $\mathrm{CBFV}$ and impaired cerebral autoregulation, are candidate cerebrovascular risk factors in patients with Anderson-Fabry disease.

\section{WHITE MATTER LESIONS IN ANDERSON-FABRY DIS- EASE}

White matter lesions (WMLs), commonly seen as hyperintensities on T2-weighted MRI scans of healthy elderly individuals, are considered to be related to small vessel disease in the brain, and are often associated with subtle cognitive and functional impairments. WMLs also show a strong correlation with a wide range of neurodegenerative and neuropsychiatric disorders. Although a number of vascular risk factors for WMLs have been identified, genetic factors are also important with twin and family studies reporting high heritability. Mutations in several genes have been described that lead to monogenic disorders manifesting WMLs, such as Fabry disease and CADASIL. Because most individuals with WMLs do not have Mendelian disorders, most of the focus has been on single nucleotide polymorphisms as genetic risk markers for WMLs, either directly or through their interactions with other genes or medical risk factors. Candidate genes examined to date include those involved in cholesterol regulation and atherosclerosis, hypertension, neuronal repair, homocysteine levels, and oxidative stress pathways. In addition, although there have been a few genome-wide linkage studies, only one genome-wide association study has been performed. The majority of the genetic findings need independent replication, and studies need to be extended to other candidate genes. Collaborative efforts to examine genome-wide associations in large samples of both sexes of a broad age range using longitudinal studies are necessary. The identification of individuals genetically at risk of developing white matter lesions will have important implications for recognizing the etiology of WMLs and thereby developing clinical intervention strategies for their prevention. Conventional MRI shows a progressive load of white matter lesions (WMLs) due to cerebral vasculopathy in the course of AFD. To quantify brain structural changes in clinically affected male and female patients with AFD, Fellgiebel A. et al. [71] performed a prospectiveDiffusion-Tensor Imaging (DTI) study in 27 adult Fabry patients $(13 \mathrm{~m}, 14 \mathrm{f})$ and 21 age-matched controls $(12 \mathrm{~m}, 9 \mathrm{f})$. Global Mean Diffusivity (MD) was increased in FD $(\mathrm{P}=0.003)$ whereas global Fractional Anisotropy (FA) did not differ significantly between FD and controls. Even FD patients without significant WMLs $(9 \mathrm{~m}, 9 \mathrm{f})$ showed increased global MD $(\mathrm{P}=0.004)$. Regions of interest with significant MD elevations were located in the frontal, parietal and temporal white matter. No differences of thalamic and hippocampal DTI measurements could be detected between FD and controls. DTI parameters did not differ between male and female patients. The data provide the first evidence of a pattern of marked structural brain tissue alterations in adult FD male and female patients even without WMLs. DTI seems to be an appropriate diagnostic tool to quantify brain tissue integrity in FD. Moreover, this method could be favorable for longitudinal assessment of brain structure alterations in FD, and for monitoring the cerebral effects of enzyme replacement therapy.

Moore et al. [72] set to determine whether elevated resting CBF in Fabry disease is primarily a cerebrovascular abnormality or is secondary to enhanced neuronal metabolism. The relationship of cerebral metabolism and blood flow to Fabry leukoencephalopathy was also investigated.

Authors measured the global and regional cerebral metabolic rate of glucose using 18-fluoro-deoxyglucose (FDG) and PET in 16 patients with Fabry disease (7 patients with leukoaraiotic lesions and 9 without) and in 7 control subjects. MRI fluid attenuated inversion recovery (FLAIR) studies were also performed in the patient and control groups. All control subjects had normal MRI FLAIR studies with no high-signal deep white matter lesions (WML). Patients were partitioned into FLAIR lesion and nonFLAIR lesion groups. Authors found no evidence of cerebral glucose hypermetabolism in Fabry disease. On the contrary, significantly decreased regional cerebral glucose metabolism (rCMRGlu) was found particularly in the deep white matter in the Fabry nonlesion group and exacerbated in the lesion group. Lesionsusceptible regions were relatively hyperperfused in non-lesion patients compared to the control group. They conclude that the elevated $\mathrm{rCBF}$ and decreased white matter rCMRGlu indicate a dissociation between metabolism and blood flow suggesting chronic deep white matter metabolic insufficiency.

Impaired kidney function is thought to be associated with small vessel disease, outcome, and mortality in the general stroke population. Data are limited regarding young patients.

Steinicke R. et al. [73] conducted a study to investigate the association of kidney function and white matter hyperintensities (WMHs) in young patients with first ischemic stroke. Authors analyzed 2500 young (18-55 years) patients with first-ever ischemic stroke from the prospective observational Stroke in Young Fabry Patients (SIFAP1) study with available MRI data on WMH. Of these, 2009 had available data concerning estimated glomerular filtration rate (eGFR). Kidney function was expressed as eGFR by the Modification of Diet in Renal Disease method. Deep WMHs on MRI were classified by the Fazekas score. Multivariate analysis was performed using a regression model with random effects. Mean eGFR was $96.7 \mathrm{~mL} / \mathrm{min}$ in those with WMH Grade 0 to 1 (none to mild), $90.7 \mathrm{~mL} / \mathrm{min}$ in WMH Grade 2 (moderate), and $89 \mathrm{~mL} / \mathrm{min}$ in WMH Grade 3 (severe). Univariate analysis revealed WMH to be associated with age $(\mathrm{P}<0.001)$, hypertension $(\mathrm{P}<0.001)$, cardiovascular disease $(\mathrm{P}=0.015)$, overweight (body mass index $>25$ $\mathrm{kg} / \mathrm{m}(2) ; \quad \mathrm{P}=0.013)$, current smoking $(\mathrm{P}=0.044)$, and eGFR $(\mathrm{P}=0.009)$. In multivariate analysis, age, hypertension, and eGFR remained associated with WMH severity. Authors concluded that in young patients with acute ischemic stroke, lower eGFR values in the normal range are associated with the presence of moderate to severe WMH. 
PSYCHIATRIC AND COGNITIVE DYSFUNCTION AND FUNCTIONAL INVOLVEMENT IN ANDERSON-FABRY DISEASE

Fabry disease affects multiple organ systems, including the central nervous system. A high proportion of patients with Fabry disease is at increased risk of developing neuropsychiatric symptoms, such as depression and neuropsychological deficits. Due to both somatic and psychological impairment, health-related quality of life (QoL) is considerably reduced in patients with Fabry disease. Although the pathophysiological mechanisms of Fabry disease have not been fully elucidated, it is surmised that sphingolipid deposits in the endothelium of small cerebral vessels lead to regional cerebral ischaemia, which may be accompanied by neuropsychiatric symptoms. Furthermore, patients with Fabry disease are chronically distressed by pain attacks and additional somatic and psychosocial impairment. The available literature on psychiatric and neuropsychological findings, psychosocial adjustment and QoL during the natural course of Fabry disease in adults is reviewed in this chapter. Psychiatric symptoms, particularly depression, are highly prevalent in men and women with Fabry disease. Although more reliable data on neuropsychological deficits in the course of Fabry disease are needed, compelling evidence is available that almost all components of QoL are impaired in affected patients. As neuropsychiatric symptoms and poor psychosocial adjustment seem to contribute to the low QoL of patients with Fabry disease, early therapeutic intervention and prevention programmes, starting in childhood or adolescence, are strongly recommended.

Literature on the neuropsychiatric and psychosocial aspects of Fabry disease reveals a paucity of reliable data. According to the available literature, depressive symptoms and disorders seem to occur frequently in males and females with Fabry disease. Depression is related to a high risk of suicide and elevated mortality, impaired psychosocial function and reduced QoL. This is consistent with other reports of depression occurring in the context of somatic illness, in which $20-50 \%$ of patients are found to suffer depressive episodes - for example, after myocardial infarction, stroke, cancer and diabetes mellitus [74,75,76]. From these studies, we have learned that co-morbid untreated depression might reduce both the prognosis of the somatic illness and the patient's QoL.

In a preliminary analysis of an ongoing study (MJ Müller and KM Müller, unpublished data), a high proportion of acutely depressed individuals (55\%) was found in a sample of 36 patients with Fabry disease, corroborating earlier observations. Additionally, about one-third of male and more than two-thirds of female patients with Fabry disease had a lifetime diagnosis of major depression according to internationally established diagnostic criteria (Diagnostic and Statistical Manual of Mental Disorders - Fourth Edition, DSM-IV). In addition, a case series has been published on four female heterozygotes with Fabry disease who had severe depression [77].

Some authors [78] investigated neuropsychological and psychiatric function in AFD patients. They studied 16 AFD patients, aged 7 to 61 years. Intelligence, language, vision-spatial abilities, memory, sensorimotor abilities, and attention and executive functions were tested with a computerized test battery as well as standard paper and pencil tests. The results were compared to known agebased norms. In addition, all patients were screened for lifelong DSM-IV Axis-I and Axis-II psychiatric diagnoses, and 4 were interviewed by a psychiatrist. Performance on most cognitive measures was within average range. All measures of information processing speed were significantly reduced, as were some measures of executive functions. Ten out of 16 patients met DSM-IV criteria for Axis I or Axis II diagnoses at some point in their lives. This preliminary study delineates a psychiatric and cognitive phenotype in AFD patients and contributes to the growing field of characterizing behavioral phenotypes of patients with genetic diseases. Authors suggest that psychiatric and neuro-psychological evaluation could be included in the patient's evaluation.

\section{PULVINAR SIGN}

Fabry disease is a multisystem $\mathrm{X}$-linked disorder characterized clinically by angiokeratoma, corneal and lenticular abnormalities, acroparesthesia, renal and cardiac dysfunction and stroke. We sought to describe novel neuroimaging characteristics of Fabry disease. Moore et al. [79] reviewed neuroradiologic records of 104 hemizygous patients with Fabry disease evaluated between 1994 and 2002. In total, $94 \mathrm{MR}$ studies consisting of T1- and T2weighted images were examined for the presence of hyperintensity on the T1-weighted images. Additional CT, gradient-echo (T2*weighted), and fat-suppression MR studies were reviewed to characterize further the $\mathrm{T} 1$ abnormality in selected patients. In some patients, cerebral blood flow (CBF) was quantified by using arterial spin tagging (AST). Overall, 22 patients (approximately 23\%) demonstrated pulvinar hyperintensity on T1-weighted images; the frequency increased with age to over $30 \%$ by age 50 years. Susceptibility-weighted $\mathrm{T} 2 *$ studies demonstrated a low-signal-intensity abnormality in the pulvinar in the more severe cases, whereas CT demonstrated the pulvinar to be mineralized. CT attenuation corresponded with an increasing signal intensity on $\mathrm{T} 1$-weighted images. Posterior circulation CBF was found to be elevated on individual AST studies, especially in the thalamus. Hyperintensity in the pulvinar on T1-weighted images is a common finding in Fabry disease, likely reflecting the presence of calcification. Although other mineralizing abnormalities may result in calcification of deep gray nuclei, exclusive involvement of the pulvinar may be distinctively characteristic to Fabry disease. Increased CBF in the posterior circulation, particularly the thalamus, suggests that the dystrophic calcification is secondary to cerebral hyperperfusion and selective vulnerability of the pulvinar and adjacent thalamic nuclei. The finding of isolated pulvinar hyperintensity on T1-weighted images should suggest Fabry disease, particularly when seen in conjunction with other nonspecific neuroradiologic manifestations of the disease (Figs. 12, 13,14).

Neuroradiological findings of AFD include ischemic stroke, white matter lesions, vascular abnormalities (vertebrobasilar dolichoectasia and vessel tortuosity), and posterior thalamus involvement (the so called pulvinar sign).
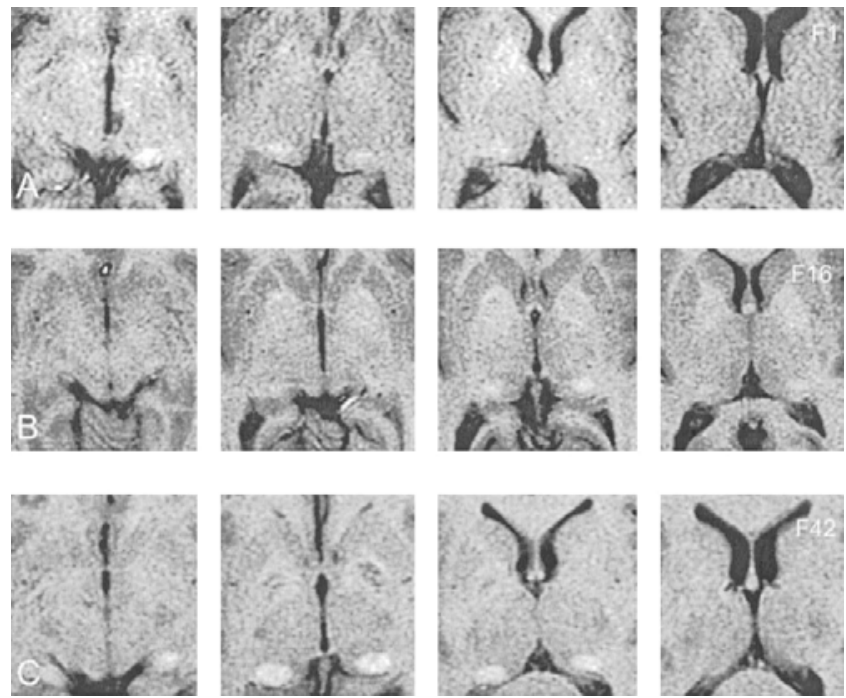

Fig. (12). Serial, axial T1-weighted MR images (5-mm sections) demonstrate the range of pulvinar hyperintensities observed.

$\mathbf{A}$ and $\mathbf{B}$, Mild to moderate abnormality. C, Marked abnormality. 

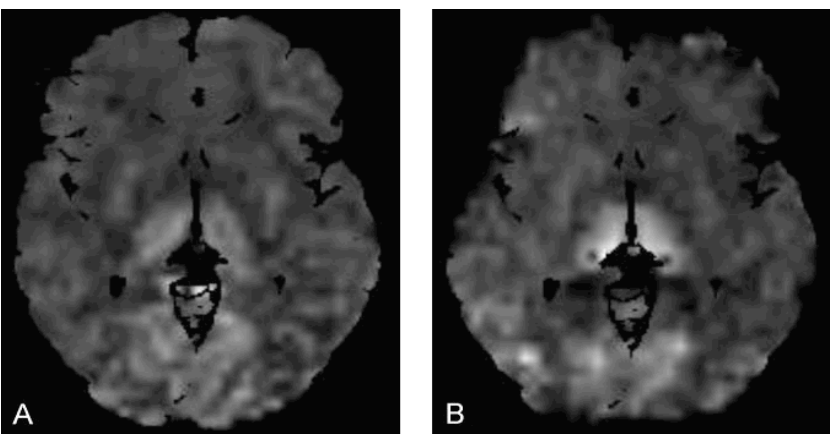

Fig. (13). Direct axial AST images demonstrateincreased relative CBF in the thalamus and posterior circulation at the anterior and posterior commissure plane in a patient without (A) and one with (B) the T1-weighted MR imaging abnormality. The dark thalamic regions in the pulvinar in B correspond to the damaged mineralized areas in the midst of the still hyperperfused pulvinar region.

A recent study [80] investigated the presence of the increased pulvinar signal intensity on T1-weighted imaging - pulvinar sign and its relationship with other clinical findings, in a non-selected cohort of Fabry patients. Authors performed a prospective analysis of two populations of patients (36 subjects) with Fabry disease. Patients were followed-up at the Department of Internal Medicine of the Bichat Hospital in Paris (France) and at the Neurological Clinic of the University Hospital of Padova (Italy). Brain MR studies of each patient included T1- and T2- weighted images, FLAIR sequences, and in some cases diffusion weighted images. A total of 36 patients (16 males, 20 females) were investigated in 14 families. The pulvinar sign was found in 5 male patients, but not in female patients. Seven patients have had at least one stroke (territorial or lacunar). There was no correlation between stroke and the pulvinar sign. All patients with the pulvinar sign had hypertrophic cardiomyopathy. Four patients out of five with the pulvinar sign were on
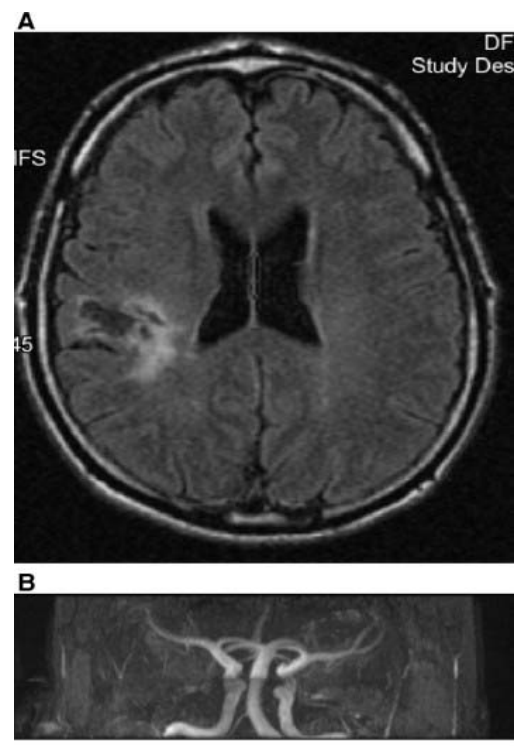

C

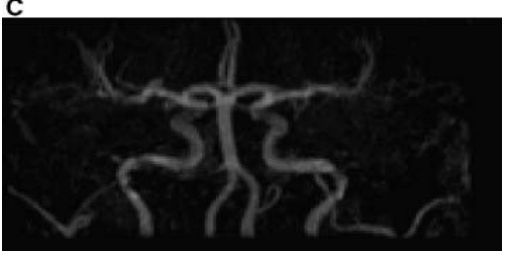

Fig. (14). A. A FLAIR MRI image of a Fabry patient with a stroke in the right middle cerebral artery territory, possibly embolic in origin. B. MR angiography (MRA) showing mild to moderate dolichoectasia of the basilar artery in a patient with Fabry disease. C. Another patient with normal appearing cerebral blood vessels on MRA. D. A FLAIR image of the patient in 2B showing a cross section of the dolichoectatic basilar artery. [From david f. moore et al. The cerebral vasculopathy of Fabry disease. Journal of the Neurological Sciences. June 2007]

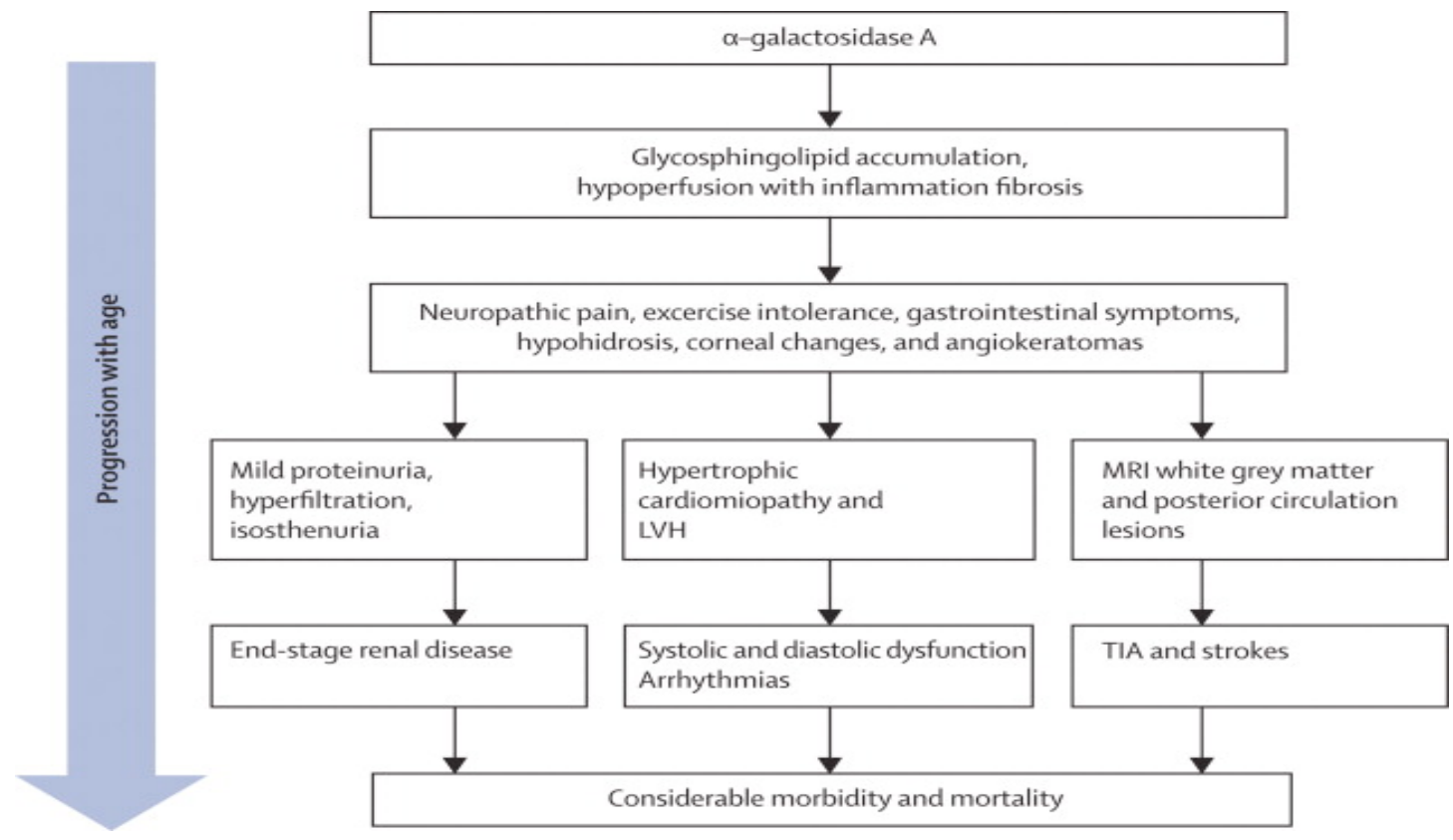

Fig. (15). Progression of clinical findings in Fabry's disease with age. Progression of any of the paths depicted can proceed independently from the others, which means that for some patients, cardiac disease will be the most severe whereas for others renal or CNS disease can predominate. LVH=left ventricular hypertrophy. TIA=transient ischaemic stroke. 

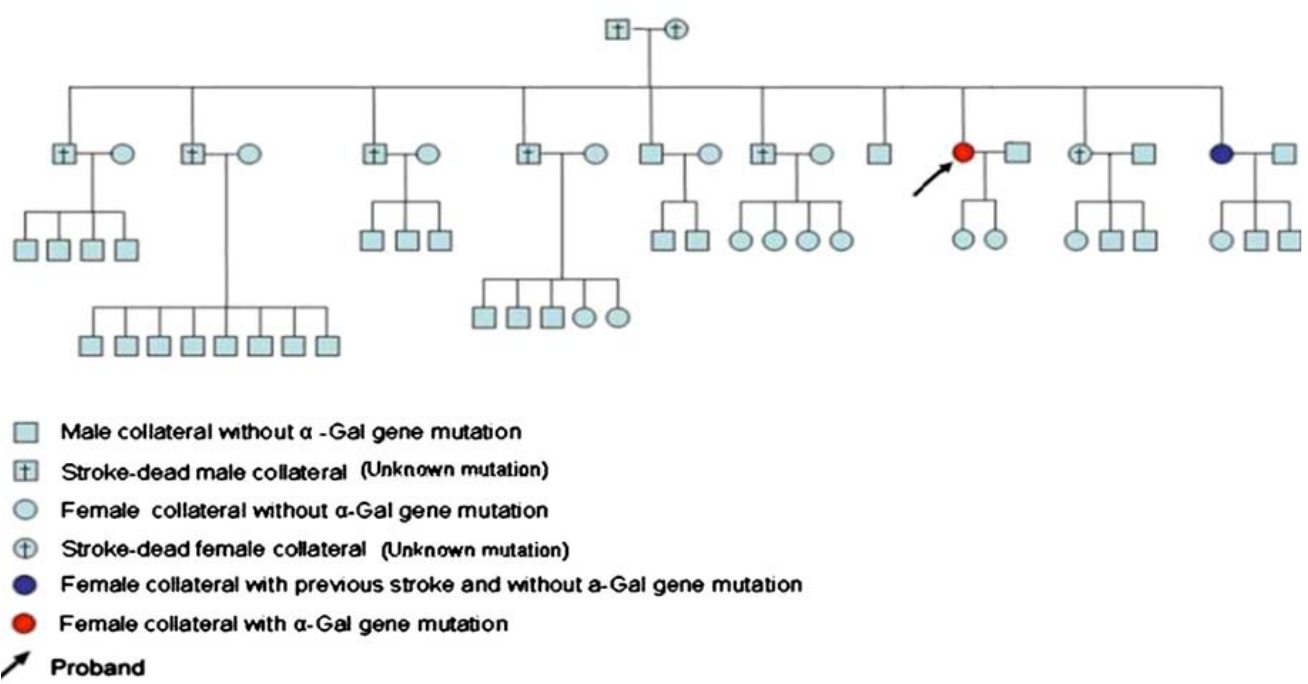

Fig. (16). Genealogic tree of patients with recurrent strokes.

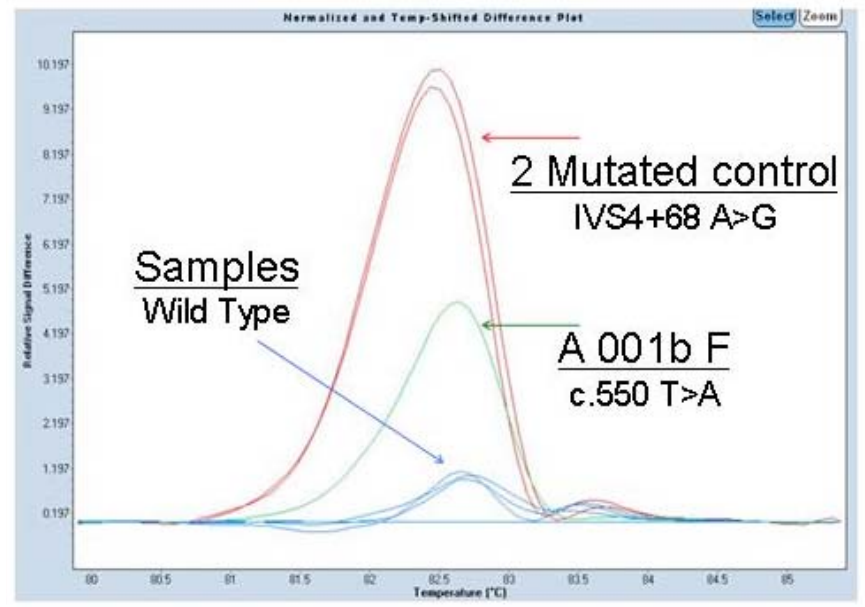

High Resolution Melting (HRM) analysis for the rapid and sensitive detection of mutations in clinical samples.

HRM assays were performed on the LightCycler ${ }^{B} \quad 480$ Real-Time PCR System (Roche Diagnostics), analyzed with the software Gene-Scanning Software Version 1.2 (Roche Diagnostics).

Gene Scanning for Exon 4 (Gene Scanning)

\begin{tabular}{|c|c|c|c|c|c|}
\hline \multicolumn{6}{|c|}{ Results } \\
\hline $\operatorname{lnc}$ & Pos. & Sample Name & Sample Type & Group & Status \\
\hline च & D1 & mutated control - woman & Unknown & mutated 1 & \\
\hline च & $\mathrm{D} 2$ & mutated control - male & Unknown & mutated 1 & \\
\hline च & $\mathrm{D} 3$ & control wild type & Melting Standard & Wt & \\
\hline ஏ & D4 & A 001b F & Unknown & mutated 2 & \\
\hline च & D5 & $\mathrm{C} 425 \mathrm{M}$ & Unknown & Wt & \\
\hline ஏ & D6 & C $426 \mathrm{M}$ & Unknown & Wt & \\
\hline च & D7 & C $431 \mathrm{~F}$ & Unknown & Wt & \\
\hline च & D8 & C $432 \mathrm{~F}$ & Unknown & Wt & \\
\hline
\end{tabular}

Fig. (17).

dialysis or had a kidney transplantation. Findings of this study suggest that the pulvinar sign is a highly specific sign of Fabry disease, found in male patients with cardiac signs and severe kidney involvement.

\section{CONCLUSIONS}

Fabry's disease is a rare hereditary lysosomal storage disease with multiorgan involvement. Deficiency of $\alpha$-galactosidase A ac- tivity leads to accumulation of neutral glycosphingolipids, especially in vascular endothelial and smooth-muscle cells (Fig. 15). Along with progressive renal and cardiac dysfunction, stroke is a major and often life-threatening burden of the disease. Cerebral vasculopathy, confirmed by neuropathological, neuroradiological, and functional studies occurs commonly and leads to ischaemic cerebrovascular events at an early age. 
However, research has shown a high prevalence of ischaemic stroke and transient ischaemic attacks, along with imaging evidence of CNS involvement, in female patients with the disease, which suggests that at least in a subgroup of clinically affected women the severity of CNS disease is comparable to that in men. Recent studies have shown a high prevalence of the disease in young patients of both sexes with cryptogenic stroke, emphasising the need for more clinical attention to be paid to this under-diagnosed disease.

We [80] recently reported a case of a 56-year-old woman with recurrent cryptogenic strokes and a familiar history of acute cerebrovascular disease (Fig. 16). Ophthalmological examination revealed whorled opacities of the cornea (cornea verticillata) and dilated tortuous conjunctival vessels. She did not show other typical signs of Fabry disease such as acroparesthesias and angiokeratoma. The patient's alpha-galactosidase A activity was $4.13 \mathrm{nmol} / \mathrm{mL} / \mathrm{h}$ in whole blood. Alpha-galactosidase A gene sequence analysis (Fig. 17) revealed a heterozygous single nucleotide point mutation at nucleotide c.550T $>\mathrm{A}$ in exon 4 in this woman, leading to the p.Tyr184Asn amino acid substitution.

Ischemic stroke is related to inflammation [82,83,84,85] and arterial stiffness [86,87] and no study had addressed this relationship in patients with AF disease and cerebrovascular disease, so this topic could represent a possible future research line.

\section{CONFLICT OF INTEREST}

The authors confirm that this article content has no conflicts of interest.

\section{ACKNOWLEDGEMENTS}

Declared none.

\section{REFERENCES}

[1] Desnick RJ, Ioannou YA, Eng CM. Alpha-galactosidase A deficiency: Fabry disease. In: Scriver CR, Beaudet al, Sly WS, Valle D (eds) The metabolic and molecular bases of inherited diseases. The Mc-Graw-Hill Companies, New York 2001, VIII ed.: pp 3733-74.

[2] MacDermot KD, Holmes A, Miners AH. Anderson-Fabry disease: clinical manifestations and impact of disease in a cohort of 60 obligate carrier females. J Med Genet 2001; 38: 769-75. 3.

[3] Whybra C, Kampmann C, Willers I, et al. Anderson-Fabry disease: Clinical manifestations of disease in female heterozygotes. J Inherit. Metab. Dis 2001; 24: 715-724. 4.

[4] Deegan PB, Baehner AF, Barba Romero MA, Hughes DA, Kampmann C, Beck M. European FOS Investigators. Natural history of Fabry disease in females in the Fabry Outcome Survey. J Med Genet 2006; 43: 347-52.

[5] Grewal RP. Stroke in Fabry's disease. J Neurol. 1994; 241: 153-6.

[6] Mitsias P, Levine S. Cerebrovascular complications of Fabry disease. Ann Neurol 1996; 40: 8-17.

[7] Mohanraj R, Leach JP, Broome JC, Smith DF. Neurological presentation of Fabry's disease in a 52 years old man. J Neurol Neurosurg Psychiatry 2002; 73: 340-2.

[8] Crutchfield KE, Patronas NJ, Dambrosia JM, et al. Quantitative analysis of cerebral vasculopathy in patients with Fabry disease. Neurology 1998; 50: 1746-9.

[9] Moore DF, Kaneski CR, Askari H, Schiffmann R. The cerebral vasculopathy of Fabry Disease. JNeurol Sci 2007; 257: 258-63.

[10] Moore DF, Altarescu G, Ling GSF, et al. Elevated cerebral blood flow velocities in Fabry disease with reversal after enzyme replacement. Stroke 2002; 33: 525-31.

[11] Hilz MJ, Kolodny EH, Brys M, Stemper B, Haendl T, Marthol H. Reduced cerebral blood flow velocity and impaired cerebral autoregulation in patients with Fabry disease. J Neurol 2004; 251: 564-70.

[12] Kaye EM, Kolodny EH, Logigian EL, Ullman MD. Nervous system involvement in Fabry's disease: clinicopathological and biochemical correlation. Ann Neurol 1988; 23: 505-9. 6.

[13] Schiffmann R, Rapkiewicz A, Abu-Asab M, et al. Pathological findings in a patient with Fabry disease who died after 2.5 years of enzyme replacement. Virchows Arch 2005; 29: 1-7.
[14] Mehta A, Ginsberg L. FOS investigators. Natural history of the cerebrovascular complications of Fabry disease. Acta Paediatr Suppl 2005; 94: 24-7.

[15] Goldstein LB, Adams R, Alberts MJ, et al. American Heart Association/American Stroke.

[16] Altarescu G, Moore DF, Schiffmann R. Effect of genetic modifiers on cerebral lesions in Fabry disease. Neurology 2005; 64: 2148-50.

[17] Ginsberg L, Manara R, Valentine AR, Kendall B, Burlina AP. Magnetic resonance imaging changes in Fabry disease. Acta Paediatrica Suppl 2006; 451: 57-62

[18] Lidove O, Klein I, Lelièvre JD, et al. Imaging Features of Fabry disease. AJR 2006; 186: 1184-19.

[19] Saposnik G, Lanthier S, Mamdani M, et al. Canadian Stroke Consortium; Stroke Outcome Research Canada (SORCan) Working Group.Fabry's disease: a prospective multicenter cohort study in young adults with cryptogenic stroke. Int J Stroke 2012; 7(3): 26573.

[20] Rolfs A, Bottcher T, Zschiesche M, et al. Prevalence of Fabry disease in patients with cryptogenic stroke: a prospective study. Lancet 2005; 366: 1794-96.

[21] Carolei A, Marini C, Ferranti E, Frontoni M, Prencipe M, Fieschi C. A prospective study of cerebral ischemia in the young. Analysis of pathogenic determinants. Stroke 1993; 24: 362-67.

[22] Kristensen B, Malm J, Carlberg B, et al. Epidemiology and etiology of ischemic stroke in young adults aged 18 to 44 years in northern Sweden. Stroke 1997; 28: 1702-09.

[23] Adams HP, Kappelle LJ, Biller J, et al. Ischemic stroke in young adults: experience in 329 patients enrolled in the Iowa Registry of stroke in young adults. Arch Neurol 1995; 52: 491-95.

[24] Sarikaya H, Yilmaz M, Michael N, Miserez AR, Steinmann B, Baumgartner RW. Zurich Fabry study - prevalence of Fabry disease in young patients with first cryptogenic ischaemic stroke or TIA. Eur J Neurol. 2012 Jun 11 [Epub ahead of print]

[25] Wozniak MA, Kittner SJ, Tuhrim S, et al. Frequency of unrecognized Fabry disease among young European-American and African-American men with first ischemic stroke. Stroke. 2010; 41(1): 78-81.

[26] Brouns R, Sheorajpanday R, Braxel E, et al. Middelheim Fabry Study (MiFaS): a retrospective Belgian study on the prevalence of Fabry disease in young patients with cryptogenic stroke. Clin Neurol Neurosurg 2007; 109: 479-84.

[27] Fellgiebel A, Muller MJ, Ginsberg L. CNS manifestations of Fabry's disease. Lancet Neurol 2006; 5: 791-5.

[28] Brouns R, Thijs V, Eyskens F, et al. BeFaS Investigators Belgian Fabry study: prevalence of Fabry disease in a cohort of 1000 young patients with cerebrovascular disease Stroke. 2010; 41(5): 863-8.

[29] Sims K, Politei J, Banikazemi M, Lee P. Stroke in Fabry disease frequently occurs before diagnosis and in the absence of other clinical events: natural history data from the Fabry Registry. Stroke. 2009; 40(3): 788-94.

[30] Fellgiebel A, Wolf DO, Kolodny E, Müller MJ. Hippocampal atrophy as a surrogate of neuronal involvement in Fabry disease. J Inherit Metab Dis 2012; 35(2): 363-7.

[31] Fellgiebel A, Keller I, Marin D, et al. Diagnostic utility of different MRI and MR angiography measures in Fabry disease. Neurology 2009; 72(1): 63-8.

[32] Fellgiebel A, Mazanek M, Whybra C, et al. Pattern of microstructural brain tissue alterations in Fabry disease: a diffusion-tensor imaging study. J Neurol 2006; 253(6): 780-7.

[33] Albrecht J, Dellani PR, Müller MJ, et al. Voxel based analyses of diffusion tensor imaging in Fabry disease. J Neurol Neurosurg Psychiatry 2007; 78(9): 964-9.

[34] Whybra C., Wendric K., Ries M., Gal A., Beck M. Clinical manifestation in female Fabry disease patients. Contrib. Nephrol 2001; 136: 245- 50. 5 .

[35] MacDermot J, MacDermot KD. Neuropathic pain in AndersonFabry disease: pathology and therapeutic options. Eur J Pharmacol 2001; 429(1-3): 121-5

[36] Scott LJ, Griffin JW, Luciano C, et al. Quantitative analysis of epidermal innervation in Fabry disease. Neurology 1999; 52 (6): 1249-54.

[37] Hilz MJ, Stemepr B, Kolodny EH. Lower limb cold exposure induces pain and prolonged small fiber dysfunction in Fabry patients. Pain 2000; 84 (2-3): 361-5. 
[38] Dutsch M, Marthol H, Stemper B, Brys M, Haendl T, Hilz M J. Small fiber dysfunction predominates in Fabry neuropathy. J Clin Neurophysiol 2002; 19 (6): 575-86.

[39] Schiffmann R, Floeter MK, Dambrosia JM, et al. Enzyme replacement therapy improves peripheral nerve and sweat function in Fabry disease. Muscle Nerve 2003; 28(6): 703-10.

[40] Moore DF, Kaneski CR, Askari H, Schiffmann R. The cerebral vasculopathy of Fabry disease, J. Neurol. Sci. 257 (2007) 258-263. R. Schiffmann. Fabry disease. Pharmacol Ther 2009; 122: 65-77.

[41] Sessa A, Meroni M, Battini G, et al. Renal pathological changes in Fabry disease, J Inherit Metab Dis 2011; 24 (Suppl. 2): 66-70.

[42] Kampmann C, Wiethoff CM, et al. The heart in Anderson Fabry disease. Z Kardiol 2002; 91: 786-95.

[43] . Desnick R.J, Brady R, Barranger J, et al. Fabry disease, an underrecognized multisystemic disorder: expert recommendations for diagnosis, management, and enzyme replacement therapy. Ann Intern Med 2003; 138: 338-46

[44] Barbey F, Brakch N, Linhart A, et al. Cardiac and vascular hypertrophy in Fabry disease: evidence for a new mechanism independent of blood pressure and glycosphingolipid deposition. Arterioscler. Thromb Vasc Biol 2006; 26: 839-44.

[45] Kalliokoski J, Kalliokoski KK, Penttinen M, et al. Structural and functional changes in peripheral vasculature of Fabry patients. J Inherit Metab Dis 2006; 29: 660-6.

[46] Heare T, Alp NJ, Priestman DA, et al. Severe endothelial dysfunction in the aorta of a mouse model of Fabry disease; partial prevention by Nbutyldeoxynojirimycin treatment. J Inherit Metab Dis 2007; 30: 79-87.

[47] Moore DF, Altarescu G, Pursley R, et al. Arterial wall properties and Womersley flow in Fabry disease. BMC Cardiovasc. Disord 2002; $2: 1$

[48] Stemper B, Hilz MJ. Postischemic cutaneous hyperperfusion in the presence of forearm hypoperfusion suggests sympathetic vasomotor dysfunction in Fabry disease. J Neurol 2003; 250: 970-6.

[49] Seino Y, Vyden JK, Philippart M, Rose HB, Nagasawa K. Peripheral hemodynamics in patients with Fabry's disease. Am. Heart J 1983; 105: 783-7.

[50] Martin L, Sturgess A, Sillence D, Murrell DF. Fabry disease in a heterozygote presenting as hand ischaemia and painful acroparaesthesia. Australas. J Dermatol 2007; 48: 40-2.

[51] Kalliokoski RJ, Kalliokoski KK, Sundell J, et al. Impaired myocardial perfusion reserve but preserved peripheral endothelial function in patients with Fabry disease. J Inherit Metab Dis 2005; 28: 56373.

[52] Dimitrow PP, Krzanowski M, Undas A. Reduced coronary flow reserve in Anderson-Fabry disease measured by transthoracic Doppler echocardiography. Cardiovasc. Ultrasound. 2005; 3.

[53] Altarescu G, Moore DF, Pursley R, et al. Enhanced endotheliumdependent vasodilation in Fabry disease. Stroke 2001; 32: 1559-62.

[54] Park JL, Whitesall SE, D'Alecy LG, Shu L, Shayman JA. Vascular dysfunction in the alpha-galactosidase A-knockout mouse is an endothelial cell-, plasma membrane-based defect. Clin Exp Pharmacol Physiol 2008; 35: 1156-63.

[55] Park JL, Shu L, Shayman JA. Differential involvement of COX1 and COX2 in the vasculopathy associated with the alphagalactosidase A-knockout mouse. Am J Physiol Heart Circ Physiol 2009; 296: H1 133-40.

[56] Hilz MJ, Kolodny EH, Brys M, Stemper B, Haendl T, Marthol H. Reduced cerebral blood flow velocity and impaired cerebral autoregulation in patients with Fabry disease. J Neurol 2004; 251: 564-70.

[57] Moore DF, Altarescu G, Ling GS, et al. Elevated cerebral blood flow velocities in Fabry disease with reversal after enzyme replacement. Stroke 2002; 33: 525-31.

[58] Hilz MJ, Kolodny EH, Brys M, Stemper B, Haendl T, Marthol H. Reduced cerebral blood flow velocity and impaired cerebral autoregulation in patients with Fabry disease. J Neurol 2004; 251: 564-70.

[59] Moore DF, Scott LT, Gladwin MT, et al. Regional cerebral hyperperfusion and nitric oxide pathway dysregulation in Fabry disease: reversal by enzyme replacement therapy. Circulation 2001; 104: 1506-12.

[60] Moore DF, Herscovitch P, Schiffmann R. Selective arterial distribution of cerebral hyperperfusion in Fabry disease. J Neuroimaging 2001; 11: 303-7.
[61] Crutchfield KE, Patronas NJ, Dambrosia JM, et al. Quantitative analysis of cerebral vasculopathy in patients with Fabry disease, Neurology 1998; 50: 1746-9. [72] R.P. Grewal, Stroke in Fabry's disease. J Neurol 1994; 241: 153-6.

[62] Kalliokoski RJ, Kalliokoski KK, Penttinen M, et al. Structural and functional changes in peripheral vasculature of Fabry patients, J Inherit Metab Dis 2006; 29: 660-6.

[63] Boutouyrie P, Laurent S, Laloux B, Lidove O, Grunfeld JP, Germain DP. Non-invasive evaluation of arterial involvement in patients affected with Fabry disease. J Med Genet 2001; 38: 629-31.

[64] Rombach SM, et al. / Molecular Genetics and Metabolism 2010; 99: 99-108 [54] P. Boutouyrie S, Laurent B, Laloux O, Lidove JP, Grunfeld DP. Germain. Arterial remodelling in Fabry disease. Acta Paediatr Suppl 2002; 91: 62-6.

[65] F. Barbey, N. Brakch, A. Linhart, et al. Cardiac and vascular hypertrophy in Fabry disease: evidence for a new mechanism independent of blood pressure and glycosphingolipid deposition. Arterioscler. Thromb. Vasc. Biol. 2006; 26: 839-44.

[66] Brakch N, Dormond O, Bekri S, et al. Evidence for a role of sphingosine-1 phosphate in cardiovascular remodelling in Fabry disease. Eur Heart J 2009. [Epub ahead of print]

[67] Aerts JM, Groener JE, Kuiper S, et al. Elevated globotriaosylsphingosine is a hallmark of Fabry disease. Proc Natl Acad Sci USA 105 (2008) 2812-7.

[68] Azevedo E, Mendes A, Seixas D, et al. Functional transcranial Doppler: presymptomatic changes in Fabry disease. Eur Neurol 2012; 67(6): 331-7.

[69] Uçeyler N, He L, Kahn AK, Breunig F, Müllges W, Sommer C. Cerebral blood flow in patients with Fabry disease as measured by Doppler sonography is not different from that in healthy individuals and is unaffected by treatment. J Ultrasound Med 2012; 31(3): 463-8.

[70] Hilz MJ, Kolodny EH, Brys M, Stemper B, Haendl T, Marthol H. Reduced cerebral blood flow velocity and impaired cerebral autoregulation in patients with Fabry disease. J Neurol 2004; 251(5): 564-70.

[71] Fellgiebel A, Mazanek M, Whybra C, et al. Pattern of microstructural brain tissue alterations in Fabry disease: a diffusion-tensor imaging study. J Neurol 2006; 253(6): 780-7.

[72] Moore DF, Altarescu G, Barker WC, Patronas NJ, Herscovitch P, Schiffmann R. White matter lesions in Fabry disease occur in 'prior' selectively hypometabolic and hyperperfused brain regions. Brain Res Bull 2003; 62(3): 231-40.

[73] Steinicke R, Gaertner B, Grittner U, et al. Kidney function and white matter disease in young stroke patients: analysis of the stroke in young fabry patients study population. Stroke 2012; 43(9): 23828

[74] Arolt V, Rothermundt M. Depressive disorders in patients with somatic illnesses. Nervenarzt 2003; 74: 1033-52.

[75] Cheok F, Schrader G, Banham D, Marker J, Hordacre AL. Identification, course, and treatment of depression after admission for a cardiac condition: rationale and patient characteristics for the Identifying Depression As a Comorbid Condition (IDACC) project. Am Heart J 2003; 146: 978-84.

[76] Cassidy E, O'Connor R, O'Keane V. Prevalence of post-stroke depression in an Irish sample and its relationship with disability and outcome following inpatient rehabilitation. Disabil Rehabil 2004; 26: 71-7.

[77] Sadek J, Shellhaas R, Camfield CS, Camfield PR, Burley J. Psychiatric findings in four female carriers of Fabry disease. Psychiatr Genet 2004; 14: 199-201.

[78] Segal P, Kohn Y, Pollak Y, Altarescu G, Galili-Weisstub E, RaasRothschild A. Psychiatric and cognitive profile in Anderson-Fabry patients: a preliminary study. J Inherit Metab Dis. 2010; 33(4): 429-36.

[79] Moore DF, Ye F, Schiffmann R, Butman JA. Increased signal intensity in the pulvinar on T1-weighted images: a pathognomonic MR imaging sign of Fabry disease. AJNR Am J Neuroradiol 2003; 24(6): 1096-101

[80] Burlina AP, Manara R, Caillaud C, et al. The pulvinar sign: frequency and clinical correlations in Fabry disease J Neurol 2008; 255(5): 738-44.

[81] Tuttolomondo A, Duro G, Miceli S, et al. Novel alphagalactosidase A mutation in a female with recurrent strokes. Clin Biochem 2012; 45(16-17): 1525-30. 
[82] Tuttolomondo A, Di Sciacca R, Di Raimondo D, et al. Neuron protection as a therapeutic target in acute ischemic stroke. Curr Top Med Chem 2009; 9(14): 1317-34.

[83] Tuttolomondo A, Pinto A, Corrao S, et al. Immuno-inflammatory and thrombotic/fibrinolytic variables associated with acute ischemic stroke diagnosis. Atherosclerosis 2009; 203(2): 503-8.

[84] Tuttolomondo A, Pinto A, Salemi G, et al. Diabetic and nondiabetic subjects with ischemic stroke: differences, subtype distribution and outcome.Nutr Metab Cardiovasc Dis 2008; 18(2): 1527.

Received: November 16, 2013
Accepted: February 15, 2013
Pinto A, Tuttolomondo A, Di Raimondo D, Fernandez P, Licata G. Risk factors profile and clinical outcome of ischemic stroke patients admitted in a Department of Internal Medicine and classified by TOAST classification. Int Angiol 2006; 25(3): 261-7.

[86] Tuttolomondo A, Di Sciacca R, Di Raimondo D, et al. Arterial stiffness indexes in acute ischemic stroke: relationship with stroke subtype. Atherosclerosis 2010; 211(1): 187-94.

[87] Tuttolomondo A, Di Raimondo D, Pecoraro R, et al. Immuneinflammatory markers and arterial stiffness indexes in subjects with acute ischemic stroke. Atherosclerosis 2010; 213(1): 311-8. 